\title{
Covalent Organic Frameworks as Electrode Materials for Rechargeable Batteries
}

\author{
Eric R. Wolfson ${ }^{a}$ (iD) \\ Erica M. Moscarello ${ }^{a}$ \\ William K. Hauga ${ }^{\text {(D) }}$ \\ Psaras L. McGrier*a (D) \\ ${ }^{a}$ Department of Chemistry and Biochemistry, The Ohio State University, Columbus, \\ Ohio 43210, USA \\ McGrier.1@osu.edu
}

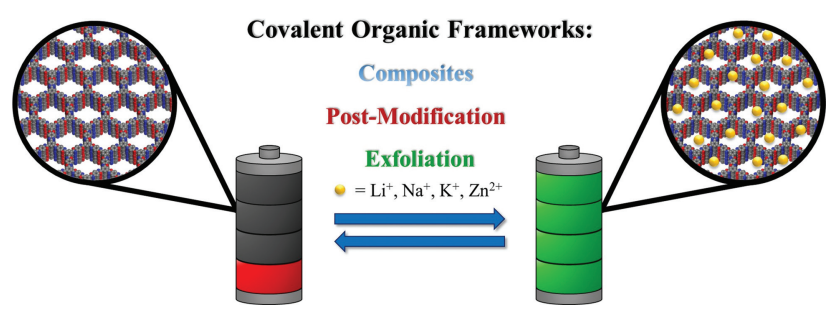

are scarce must be addressed. The storage of energy produced via renewable sources is therefore of great importance as a potential solution. As such, research into high-performance energy-storage systems with high capacities (generally reported as $\mathrm{mA} \mathrm{h} \mathrm{g}^{-1}$ ), high coulombic efficiencies (generally reported as percentages), and long cycling lifespans has become a top priority for scientists.

Lithium-ion batteries (LIBs) have become ubiquitous energy-storage devices due to their high energy densities, attractive redox potentials, high coulombic efficiencies, and fast charge mobility. These features have led to a diverse range of rechargeable battery applications through careful design of the electrode materials. ${ }^{1}$ However, the demand for LIBs with higher energy-storage capacities and longer cycling lifetimes has spurred continuing development of novel materials such as inorganic Ni-rich systems and high voltage spinel systems. ${ }^{2,3}$ While these inorganic materials are capable of excellent performance in many energystorage applications, they often suffer from critical issues such as irreversible capacity loss during initial cycles, capacity fading at high rates, and electrode degradation. In addition, because inorganic electrodes often require heavy and expensive metals, there is much room for improvement in the realm of energy density and production cost for commercial use.

Organic-based electrode materials (OBEMs) are a promising candidate for state-of-the-art solutions to the energy-storage problem. ${ }^{4}$ In contrast to their inorganic counterparts, OBEMs are comprised mainly of lighter $\mathrm{C}, \mathrm{H}$, $\mathrm{N}, \mathrm{O}$, and $\mathrm{S}$ atoms, resulting in less dense and more costeffective electrodes. ${ }^{5}$ In addition, there is an exceptionally large degree of variability possible when designing OBEMs. The ability to incorporate well-studied, redox-active building blocks into a bulk structure has led to an extensive library of materials and deeper fundamental understanding of structure-function relationships. Furthermore, by taking advantage of the flexibility inherent in some covalentlylinked systems, electrodes capable of performing well even while stretched or bent have been reported. ${ }^{6-8}$ However, despite their often highly conjugated systems, OBEMs can suffer from low electrical conductivity, requiring the 
Biosketches

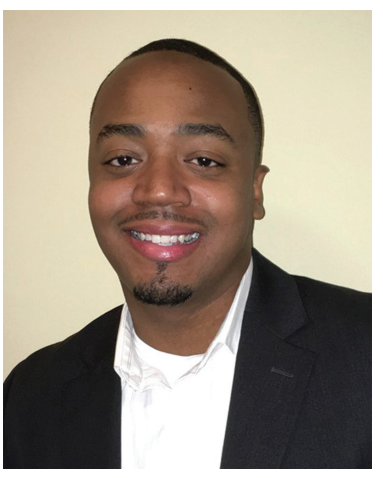

Psaras is an Associate Professor in the Department of Chemistry and Biochemistry at The Ohio State University. He received his BS in Chemistry from the University of South Carolina-Aiken in 2004 and his PhD in Organic/Polymer chemistry from the Georgia Institute of Technology (Georgia Tech) in 2010 under the

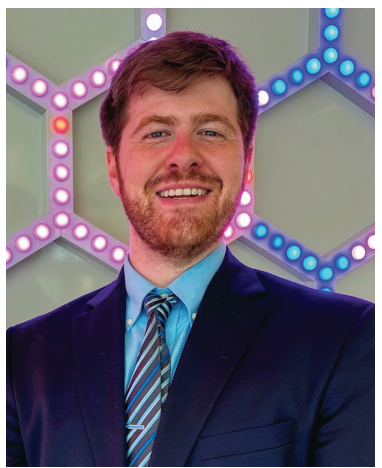

Eric Wolfson received his bachelor's degree in Chemistry in 2015 from University of Maryland Baltimore County. He has con-

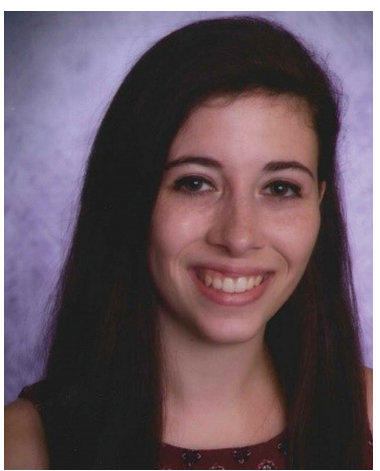

Erica Moscarello received her BS from Rowan University in 2017, where she worked under the supervision of Professor Gustavo Moura-Letts. She

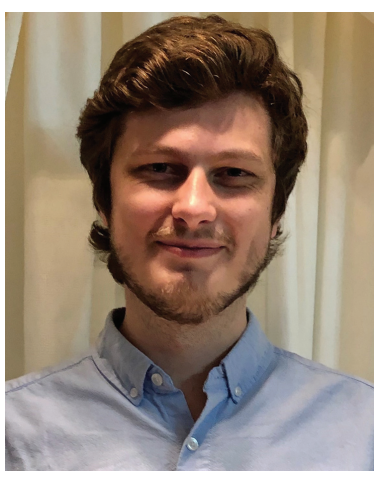

W. Karl Haug is a PhD candidate advised by Psaras L. McGrier at The Ohio State University. He received his bachelor's degree from University of Denver in 2016 where he worked on direction of Professor Uwe Bunz. After finishing his $\mathrm{PhD}$, he moved to Northwestern University (2010-2013) to join the group of Professor Sir Fraser Stoddart as a postdoctoral scholar on a fellowship from Georgia Tech Facilitating Academic Careers in Engineering and Science (FACES) committee and the National Science
Foundation (NSF). His current research interests include the synthesis and utilization of 2D and 3D porous polymers for practical applications. $\mathrm{He}$ is a recipient of the 2018 ACS Division of Polymeric Materials: Science and Engineering (PMSE) Young Investigator Award. ducted his $\mathrm{PhD}$ research under the guidance of Psaras McGrier since 2016 at The Ohio State University. As a doctoral student, his work focuses on functional porous organic polymers for use as cutting-edge energy-storage materials. is currently in the fourth year of her $\mathrm{PhD}$ at The Ohio State University under the supervision of Professor Psaras McGrier. As a doctoral stu- dent, her work focuses on investigating and tuning the properties of oxazole-linked covalent organic frameworks. photo-induced cyclizations of orthooxaxylylenes, in the lab of Andrei Kutateladze. His current research interests are focused on the utilization of metal-doped covalent organic frameworks as platforms for recyclable and sustainable heterogeneous catalysis. 
addition of conductive dopants, which decrease the battery's overall energy density. Decreases in performance have also been linked to dissolution of conductive polymers into their surrounding organic electrolyte. ${ }^{9,10}$

Covalent organic frameworks (COFs) are an advanced class of crystalline porous polymers that enable the integration of various building blocks with molecular precision. ${ }^{11,12}$ The modular nature of COFs has made them attractive for applications related to catalysis, ${ }^{13-15}$ gas storage, ${ }^{16}$ and optoelectronics. ${ }^{17}$

Much of the early COF research focused on leveraging symmetry and structure-function relationships to obtain high-performance functional materials. A large area of research has since been devoted to developing methods to incorporate novel linkages and building blocks, allowing for a diverse range of COF architectures. From this diversity arose COFs that incorporate redox-active monomers for energyrelated applications including photovoltaics ${ }^{18}$ and supercapacitors. ${ }^{19}$ Due to their robust stabilities, insolubility in electrolyte solutions, and high solvent permeability arising from their ordered periodic structures, COFs are particularly attractive candidates for the development of novel electrode materials for energy storage. Additionally, the large degree of $\pi$-conjugation throughout COF structures has generated interest in processing them into composite materials for enhanced performance.

The following review will discuss COFs in the context of their electrochemical energy-storage properties. Specifically, this review will include a summary of methods used to fabricate redox-active COFs, a collection of COFs with known energy-storage properties, and a discussion about factors that have proven critical to producing high-performing COF-based electrode materials. While most of the information provided herein will focus on LIB technology, a portion of the review will be dedicated to COF-based electrode materials beyond traditional LIBs. Finally, the challenges and outlook of the field will be discussed.

\section{Working Principles of COF-Based Electrodes}

When utilizing COFs as electrode materials for secondary batteries, the charge storage process is dependent on the redox-active components of the material, which will generally place them into one of two types of organic electrode materials: p-type or n-type. During the process of LIB cycling, p-type materials undergo oxidation to a positively charged state, which is balanced by the release of cations to the counter electrode. Conversely, n-type organic electrode materials undergo reduction to a negatively charged state, attracting cations which are then stored (Figure 1). The difference in redox potentials between the anode and the cathode serves as the thermodynamic gradient for the discharge process. The subsequent charging
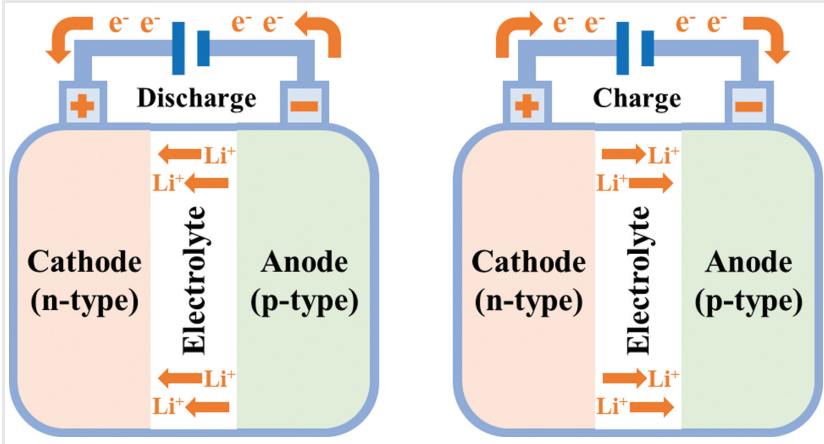

Figure 1 Schematic representation of Li-ion batteries.

is accomplished by using electrical energy to reverse the process. Therefore, a high-performing electrode material is one that has suitable redox potentials for its counter electrode, highly reversible redox chemistry, fast charge kinetics, and a large number of active sites. All of these criteria must be addressed by an electrode material that is stable and insoluble to prevent dissolution into the electrolyte while the battery cycles. Taken together, fulfilling these conditions yields batteries with long cycling lifetimes for high-capacity or high-power applications.

Currently, traditional LIBs are the frontrunner in commercial battery technology due to lithium's low redox potential ( $-3.05 \mathrm{~V}$ vs. standard hydrogen electrode), light atomic mass (6.94 $\mathrm{g} \mathrm{mol}^{-1}$ ), and small atomic radius (90 pm). These unique properties have made it amenable to either high-power or high-energy applications, depending on the components used to assemble the battery cell. However, limitations in both performance and safety have spurred interest in next-generation, nontraditional $\mathrm{Li}$-ion technologies such as Li-sulfur (Li-S), Li-air, and solid-state batteries. ${ }^{20}$ Many of these systems benefit not only from the high performance possible from leveraging lithium's inherent properties, but also from lower costs and toxicities in comparison to the widely commercialized LIBs (e.g., $\mathrm{LiCoO}_{2}$ ). Unfortunately, in spite of the advantages Li-ion technology provides, lithium's low earth abundance $(0.0017 \%)$ and poorly efficient battery recycling methods severely limit the longterm application of LIBs. As a result, other more earthabundant ion sources such as sodium, potassium, and zinc are being investigated for battery technology and will be discussed in detail later in the review.

\section{General COF Synthesis and Design Principles}

The design of COFs is based on utilizing dynamic covalent chemistry in conjunction with noncovalent interactions to yield permanently porous, ordered materials. In addition, utilizing only organic components for COFs can produce materials with exceptionally low densities ${ }^{21}$ and high thermal 
stabilities. $^{22}$ Originally synthesized in 2005, Yaghi and coworkers employed highly reversible boronate ester and boroxine condensation reactions to develop the first reported COFs. ${ }^{12}$ Unfortunately, the Lewis acidic nature of boron led to issues with hydrolytic stability. Consequently, interest in more chemically robust frameworks led to the development of more stable linkages including azines, ${ }^{23,24}$ oxazoles, ${ }^{25-27}$ and olefins (Figure 2). ${ }^{28-30}$

By leveraging the design principles mentioned above, it has become possible to obtain a rich variety of twodimensional (2D) and three-dimensional (3D) COF architectures through careful selection of $\pi$-electron-rich monomers with different symmetries (Figure 3). Indeed, it has allowed molecular-scale, topological precision while simultaneously providing strategies for synthesis of functional materials by leveraging structure-function relationships. Incorporating monomers with known functionalities has proven to yield COFs with predictable bulk properties for use in specific applications where small-molecule systems may be insufficient. Additionally, the inclusion of functional groups that remain unreactive during COF synthesis provides a route for postsynthetic modification of the bulk material. Taken together, these design strategies have provided a vast assortment of functional COFs for a growing library of applications.

Initially, COFs were synthesized through solvothermal methods in order to encourage reversibility and the eventual formation of a thermodynamically favored product. However, the microcrystalline powders produced via this method typically limited the application of COFs to those where a bulk powder is sufficient. Interest in addressing this challenge produced new synthesis and processing methodologies that provided COFs in a variety of forms including membranes, ${ }^{31-33}$ monoliths, ${ }^{34-37}$ and exfoliated sheets. ${ }^{38-40}$ Additionally, COFs have proven amenable to processing as composite materials, which work synergistically to further enhance performance.

\section{Synthesis and Design of COFs for OBEM Applications}

On rare occasions, the design methods mentioned above have been successfully employed to yield COFs that perform well as electrode materials for LIBs in their pristine, bulk form. One such case was reported by $\mathrm{Zhou}, \mathrm{Bu}$, and coworkers where a naphthalimide-based COF was used as a cathode material for LIBs. ${ }^{41}$ Combining 2,7-diaminobenzo [lmn][3,8]phenanthroline-1,3,6,8(2H,7H)-tetraone (DANT) with triformylphloroglucinol (Tp) yielded Tp-DANT-COF (Figure 4a). After assembling into LIB coin cells, the cycling performance for Tp-DANT-COF was tested, revealing initial discharge and charge capacities of 93.4 and $78.9 \mathrm{~mA} \mathrm{~h} \mathrm{~g}^{-1}$, respectively, at a current loading of $1.5 \mathrm{C}$. The initial capacity loss was contributed to the decomposition of the electrolyte. The cells showed good cycling stability over 200 cycles, with coulombic efficiencies remaining above $95 \%$ for the majority of cycling (Figure $4 \mathrm{~b}$ ). The rate performance was also assessed at rates of $0.37,0.75,1.5,3.7,7.5$, and 14.9 C, which showed reversible capacities of $104.3,96.7$, 92.3, 86.0, 79.2, and $66.3 \mathrm{~mA} \mathrm{~h} \mathrm{~g}^{-1}$, respectively. Returning the current loading to initial conditions yielded comparable reversible capacity values, demonstrating the stability of the material under intense redox conditions.

To determine the effect of the Tp linker on the material's performance, 1,3,5-triformylbenzene $(\mathrm{Tb})$ was combined with DANT to form Tb-DANT-COF (Figure 4a). While the cyclic voltammetry (CV) profile was similar (Figure 4d, e), the authors note a decrease in the gap between the oxidation and reduction potentials in comparison to Tp-DANT-COF, which is attributed to changes in the overall conjugation of the structure. Galvanostatic charge/discharge cycling of Tb-DANT-COF produced a reversible capacity of $123.7 \mathrm{~mA} \mathrm{~h} \mathrm{~g}^{-1}$ at a rate of $1.4 \mathrm{C}$. (Figure $4 \mathrm{c}$ ). Notably, DANT, Tp, and Tb could not be reliably used as organic electrode materials on their own due to their solubility in the battery electrolyte, demonstrating the importance of controlling solubility via bulk structures.

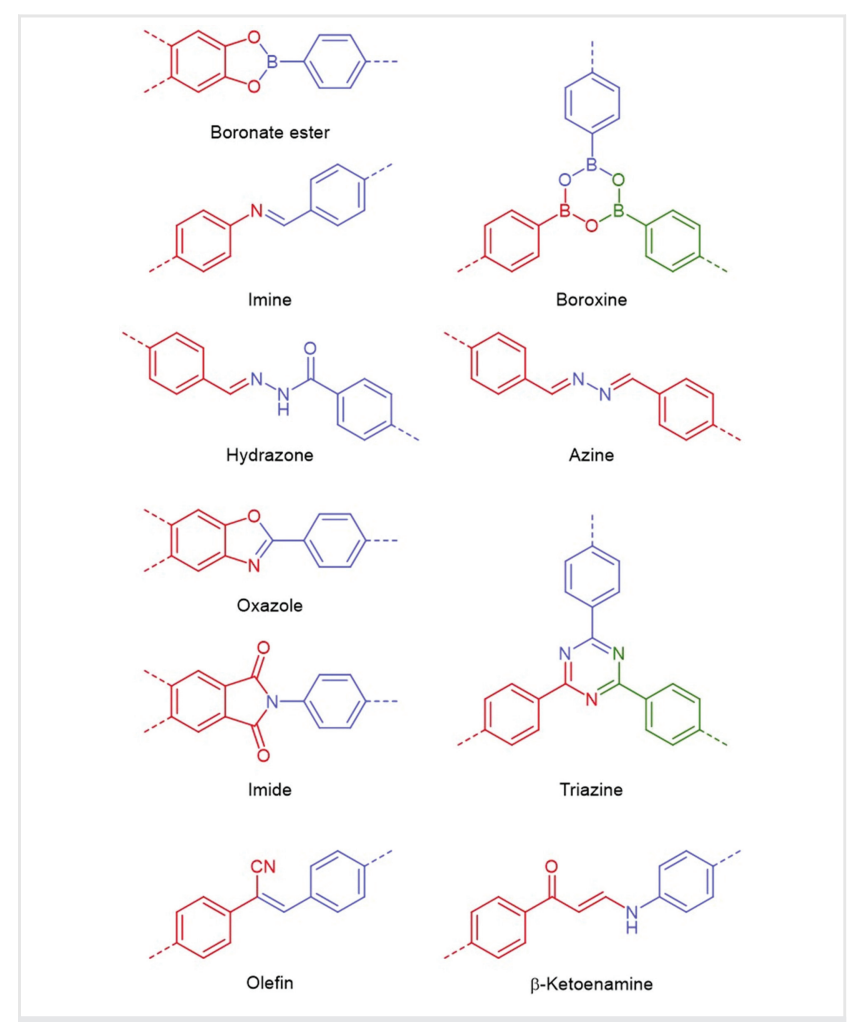

Figure 2 Common linkages used in COF synthesis. 


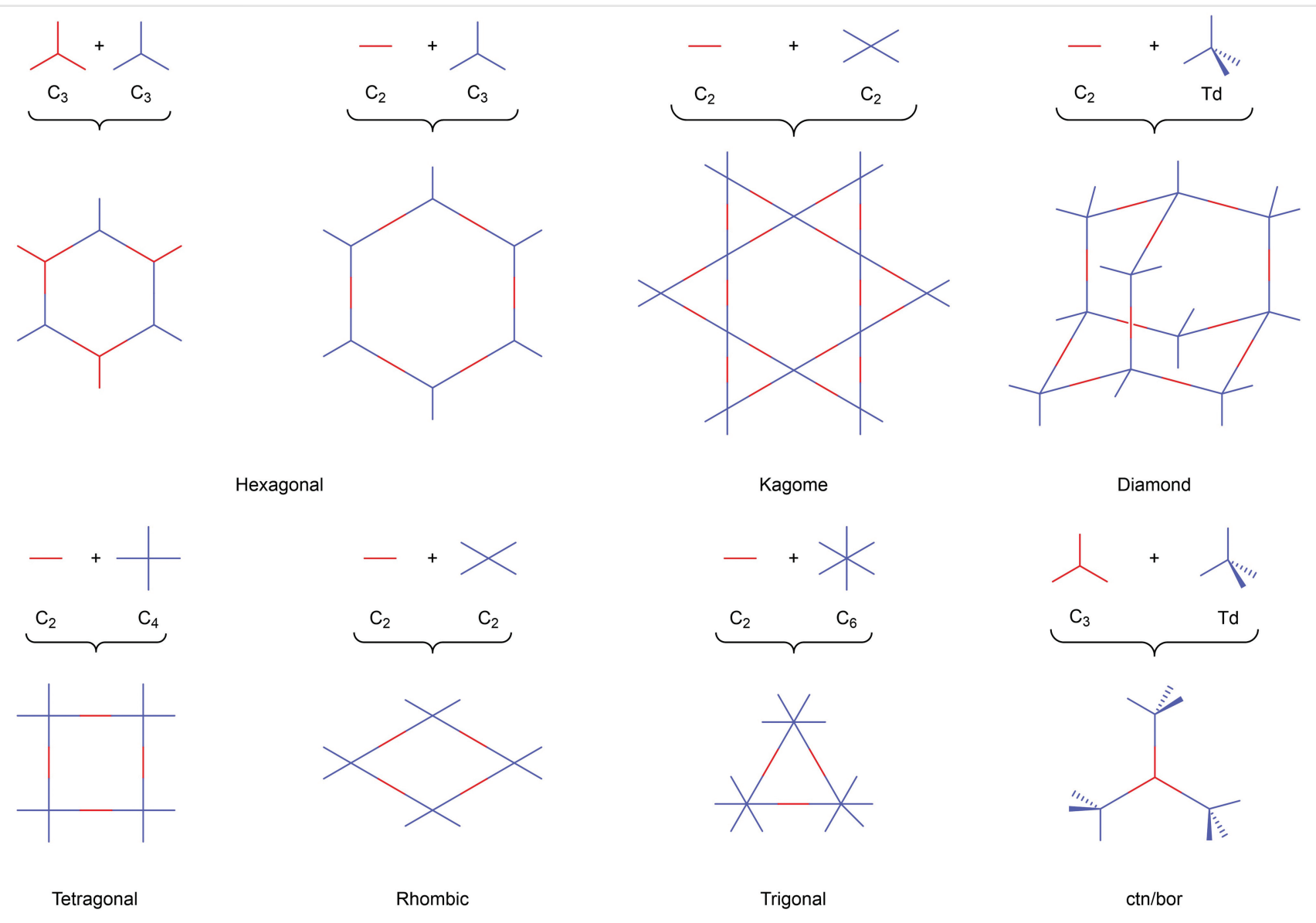

Figure 3 Common topologies accessible through COF synthesis.

In addition to naphthalimides, it has been shown that dehydrobenzo[12]annulene (DBA) is capable of reversible redox behavior at low potentials. McGrier and coworkers utilized an aldehyde-functionalized DBA and p-phenylenediamine (PPD) to synthesize highly crystalline DBA-COF 3 (Figure 5a), which was then used as an anode material for LIBs. $^{42}$ After 90 cycles at a current loading of $50 \mathrm{~mA} \mathrm{~g}^{-1}$, DBA-COF 3 displayed a reversible capacity of $207 \mathrm{~mA} \mathrm{~h} \mathrm{~g}^{-1}$ and a coulombic efficiency that remained over $95 \%$ for the majority of cycling (Figure 5b, c). Rate performance studies yielded capacities of 184, 149, 123, 107 , and $90 \mathrm{~mA} \mathrm{~h} \mathrm{~g}^{-1}$ when cycled at 50, 100, 250, 500, and $1000 \mathrm{~mA} \mathrm{~g}^{-1}$ current loadings, respectively (Figure 5d). Notably, when current loadings were returned to $50 \mathrm{~mA} \mathrm{~g}^{-1}$, the reversible capacity returned to comparable levels as well, demonstrating the excellent rate stability of the material. X-ray photoelectron spectroscopy (XPS) showed clear attenuation and regrowth of the alkyne peak after lithiation/delithiation, demonstrating the critical role of the DBA alkyne moieties in DBA-COF 3's performance.

\section{Enhancing Performance via Sulfur Impregnation}

$\mathrm{Li}-\mathrm{S}$ batteries have been of interest in recent years in the drive to build batteries with higher capacities in comparison to LIBs. Li-S batteries have a high theoretical capacity of $1672 \mathrm{~mA} \mathrm{~h} \mathrm{~g}^{-1}$. In addition, sulfur has the added benefits of being naturally abundant as well as environmentally and economically friendly. ${ }^{43-45}$ Two obstacles Li-S batteries face are their electronic insulating behavior and low cyclability as a consequence of the loss of soluble lithium polysulfide (PS) intermediates generated during the charge/discharge process. $^{46}$ To bypass this issue, there has been research into utilizing nano- or mesoporous materials such as metalorganic frameworks, ${ }^{47-49}$ porous carbon, ${ }^{50-53}$ and more recently, COFs for sulfur impregnation. ${ }^{44}$

Wang and coworkers have sought to minimize the issue of low cyclability by impregnating sulfur into the pores of COFs to effectively trap the generated lithium PSs. ${ }^{54} \mathrm{~A}$ covalent triazine-based framework (CTF), CTF-1, was chosen for its semiconductive properties as well as its 
a)

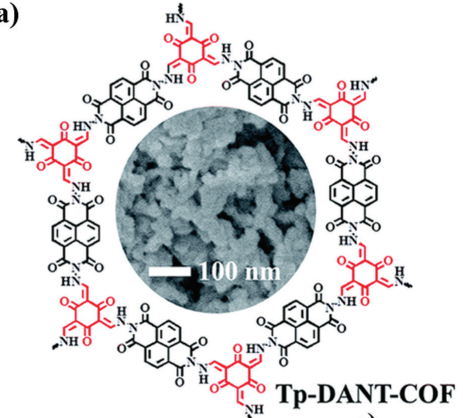

b)

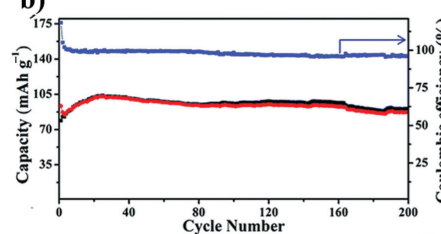

c)

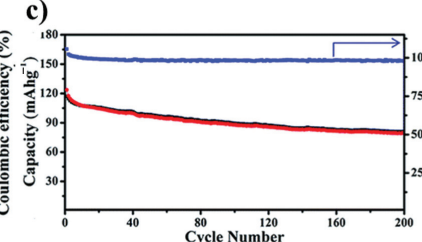

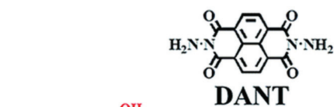

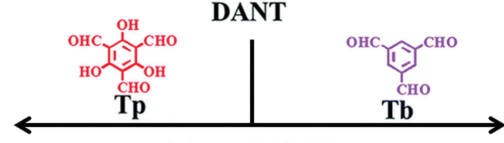

Solvents under $\mathrm{Ar}$ $120{ }^{\circ} \mathrm{C}, 120 \mathrm{~h}$

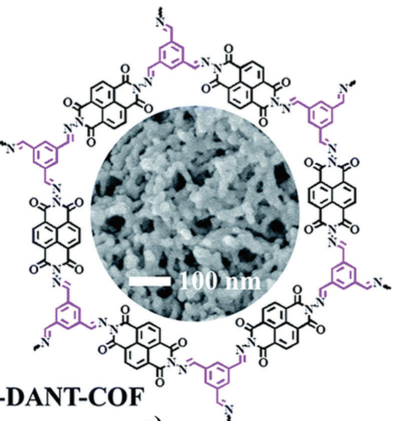

d)

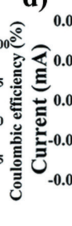

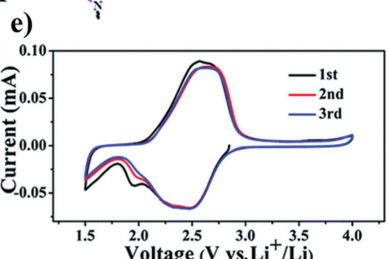

Figure 4 (a) Synthesis of Tp-DANT-COF (left) and Tb-DANT-COF (right). Cycling performance of (b) Tp-DANT-COF at 1.5 C and (c) Tb-DANT-COF at $1.4 \mathrm{C}$ (red: discharge; black: charge; blue: coulombic efficiency). CV curves of (d) Tp-DANT-COF and (e) Tb-DANT-COF at a scan rate of $0.1 \mathrm{mV} \mathrm{s}^{-1}$. Adapted with permission from Ref. 41. Copyright 2016 The Royal Society of Chemistry.

a)
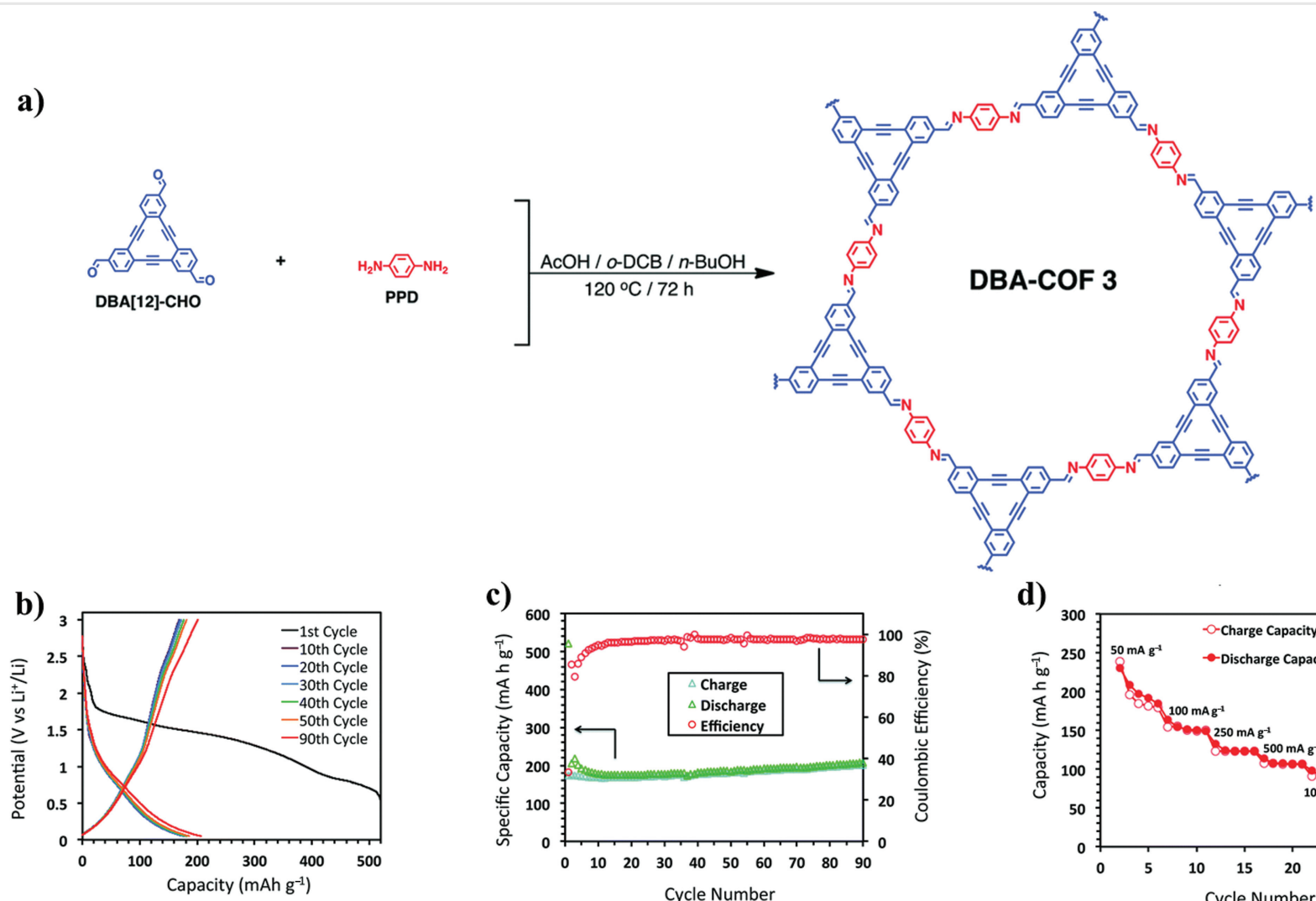

c)

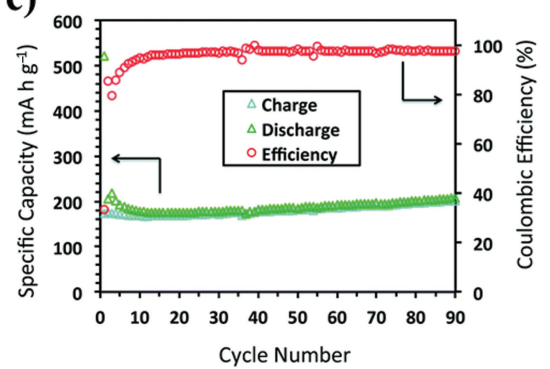

d)

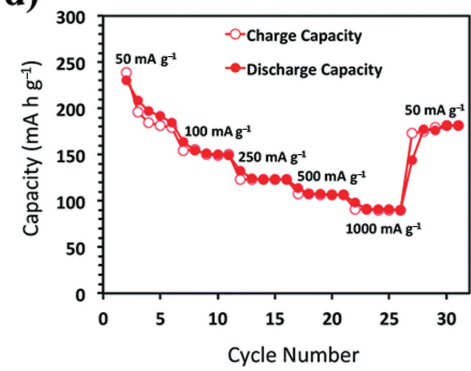

Figure 5 (a) Synthesis of DBA-COF 3. (b) Charge/discharge profile at $50 \mathrm{~mA} \mathrm{~g}^{-1}$. (c) Cycling performance after 90 cycles at $50 \mathrm{~mA} \mathrm{~g}^{-1}$. (d) Capacities of DBA-COF 3 at different charge/discharge rates ranging from 50 to $1000 \mathrm{~mA} \mathrm{~g}^{-1}$ at 5 cycles each. Adapted with permission from Ref. 42 . Copyright 2020 The Royal Society of Chemistry. 
high thermal and chemical stability. CTF-1 was synthesized via the cyclotrimerization of 1,4-dicyanobenzene with $\mathrm{ZnCl}_{2}$. It was noted that the small pore size of CTF-1 $(1.23 \mathrm{~nm})$ should be optimal for the restricted aggregation of $\mathrm{S}_{8}$ in the channels. A melt-diffusion technique was used to prepare the CTF- $1 / \mathrm{S} @ 155^{\circ} \mathrm{C}$ composite with a CTF-1:S weight ratio of $3: 2$ (Figure $6 a$ ). The occupation of sulfur in the pores was confirmed by the decrease in both surface area from 789 to $1.6 \mathrm{~m}^{2} \mathrm{~g}^{-1}$ and pore size volume from 0.37 to $0.0036 \mathrm{~cm}^{3} \mathrm{~g}^{-1}$. Elemental sulfur mapping also showed the homogeneous distribution of sulfur throughout the material (Figure 6b). The constructed coin cell battery showed a capacity of $1197 \mathrm{~mA} \mathrm{~h} \mathrm{~g}^{-1}$ at $0.1 \mathrm{C}$ at the second cycle, which dropped to $982 \mathrm{~mA} \mathrm{~h} \mathrm{~g}^{-1}$ after the 20th cycle and to $762 \mathrm{~mA} \mathrm{~h} \mathrm{~g}^{-1}$ after the 50th cycle. A coulombic efficiency of $97 \%$ was maintained indicating the strong absorption of $\mathrm{S}_{8}$ in the pores inhibiting the leaching of lithium PS intermediates. In comparison, CTF-1/S@RT exhibited a lower initial capacity of $1015 \mathrm{~mA} \mathrm{~h} \mathrm{~g} \mathrm{~g}^{-1}$ decreasing to $480 \mathrm{~mA} \mathrm{~h} \mathrm{~g}^{-1}$ after 20 cycles (Figure 6c). This low cycling stability was attributed to the fact that the composite prepared at room temperature did not allow for the embedding of sulfur into the material's pores. Wang and coworkers found these results promising and turned their focus on designing a COF more suited for sulfur storage.

After embedding sulfur into the pores of CTF-1 in 2014, Wang and coworkers sought to design a porphyrin-based COF for sulfur storage in hopes of an improved performance. ${ }^{55}$ PorCOF was designed and synthesized via the condensation reaction between PPD and 5,10,15,20-tetrakis(4-benzaldehyde)porphyrin as a host material for sulfur (Figure 7a). The large pore volume $\left(0.71 \mathrm{~cm}^{3} \mathrm{~g}^{-1}\right)$ and the narrow pore size distribution of Por-COF were cited as positive attributes for acting as an appropriate sulfur-storage material. A meltdiffusion procedure was utilized to synthesize the Por-COF/S composite with a Por-COF:S weight ratio of 45:55 (Figure 7b), confirmed by thermogravimetric analysis (TGA). The successful diffusion of sulfur into the pores of the COF was confirmed by the decrease in pore volume from 0.71 to $0.037 \mathrm{~cm}^{3} \mathrm{~g}^{-1}$. Once utilized in a battery, after 200 cycles at $0.5 \mathrm{C}$ a reversible

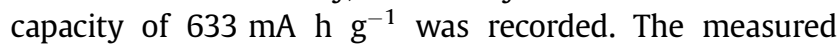
discharge capacity was $1166 \mathrm{~mA} \mathrm{~h} \mathrm{~g}^{-1}$ after the 1 st cycle, dropping to $929 \mathrm{~mA} \mathrm{~h} \mathrm{~g}^{-1}$ after the second and leveled off at $870 \mathrm{~mA} \mathrm{~h} \mathrm{~g}^{-1}$. This capacity drop in the early cycles was attributed to sulfur residing on the surface of the COF which was then reduced during the cathodic cycle and dissolved into the electrolyte. XPS analysis on the cathode indicated the generation of $\mathrm{Li}_{2} \mathrm{~S}_{2}$ and $\mathrm{Li}_{2} \mathrm{~S}_{8}$ in the charge/discharge process leading to low coulombic efficiencies.

Wang and coworkers used a 2D pyrene-based COF (Py-COF) in 2018 as a host material for sulfur to employ in Li-S batteries (Figure 8a). ${ }^{56}$ The Py-COF/S composite was formed via a melt-diffusion procedure using a Py-COF:S weight ratio of 30:70. After this process, a decrease in a)
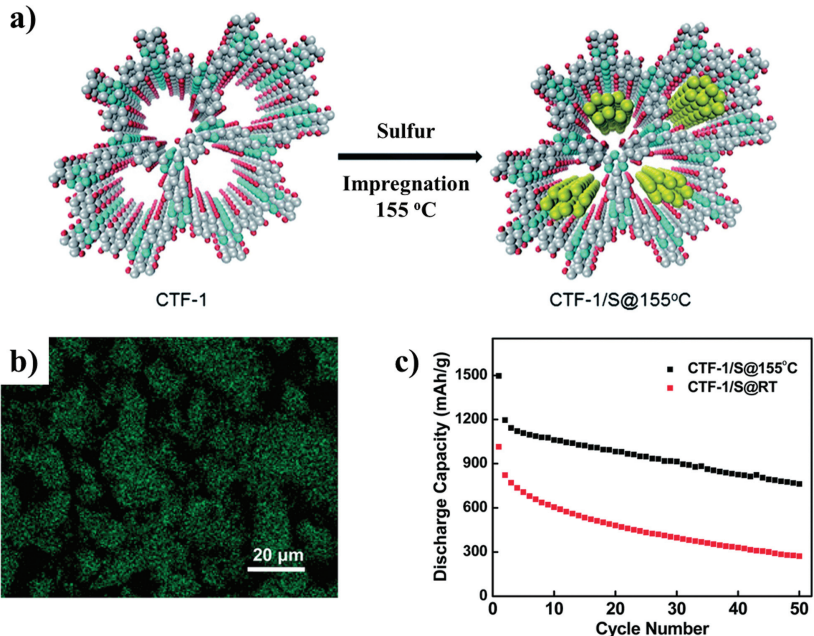

Figure 6 (a) Schematic representation of the sulfur impregnation of CTF1 to generate CTF-1/S (grey: carbon; blue: nitrogen; red: hydrogen; yellow: sulfur). (b) Sulfur elemental mapping of CTF-1/S@155 ${ }^{\circ} \mathrm{C}$. (c) Cycling performance of CTF-1/S@155 ${ }^{\circ} \mathrm{C}$ and CTF-1/S@RT at 0.1 C. Adapted with permission from Ref. 54. Copyright 2014 The Royal Society of Chemistry.

surface area from 2093 to $10 \mathrm{~m}^{2} \mathrm{~g}^{-1}$ and a decrease in pore volume from 1.25 to $0.07 \mathrm{~cm}^{3} \mathrm{~g}^{-1}$ were noted, indicating the presence of sulfur trapped in the COF pores, which was further confirmed by the homogeneous distribution of sulfur seen in energy dispersive spectroscopy. When utilized as a cathode, Py-COF delivered a reversible capacity of $963.4 \mathrm{~mA} \mathrm{~h} \mathrm{~g}^{-1}$ over 100 cycles at $1 \mathrm{C}$. As a control, $\mathrm{Py}-\mathrm{COF} / \mathrm{S}$ was directly compared to a commercially available microporous carbon, BP2000, which was functionalized into a S-containing composite referred to as BP2000/S. After 550 cycles at $5 \mathrm{C}$, a reversible capacity of $481.2 \mathrm{~mA} \mathrm{~h} \mathrm{~g}^{-1}$ could be achieved by the $\mathrm{Py}-\mathrm{COF} / \mathrm{S}$ composite. In contrast, the BP2000/S electrode exhibited a lower reversible capacity of $265 \mathrm{~mA} \mathrm{~h} \mathrm{~g}^{-1}$ at $5 \mathrm{C}$ after just 220 cycles (Figure $8 \mathrm{~b}$ ). These results show that utilizing $2 \mathrm{D}$ COFs for $\mathrm{Li}-\mathrm{S}$ based batteries can produce electrodes with large high-rate capacities as well as long-term stabilities. CV measurements were performed to further understand the high electrochemical performance of the Py-COF/S electrode. It was concluded that the rates of $\mathrm{Li}$-ion diffusion in the $\mathrm{Py}-\mathrm{COF} / \mathrm{S}$ electrode are much faster than those in BP2000/S, most likely as a consequence of the one-dimensional (1D) channels in Py-COF acting as diffusion pathways.

\section{Enhancing Performance via Polysulfide Trapping}

Most challenges facing $\mathrm{Li}-\mathrm{S}$ batteries involve the formation of PSs during the activation of the cathode and the shuttle effect in which generated long-chain PSs dissolve into the electrolyte and diffuse to the lithium anode where they are 
a)

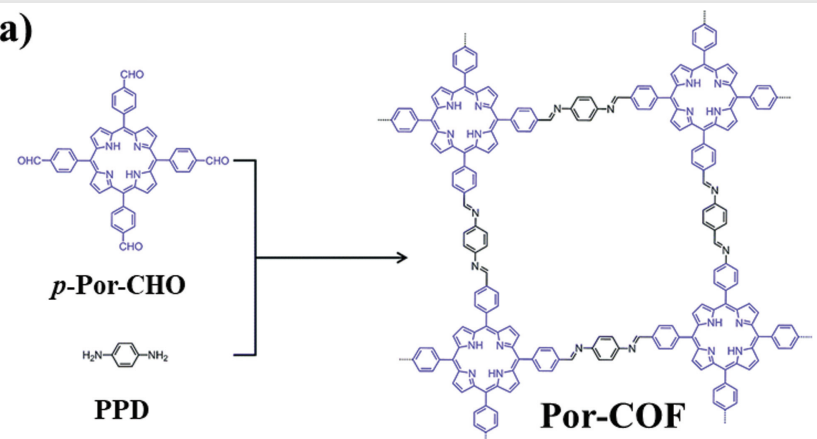

b)

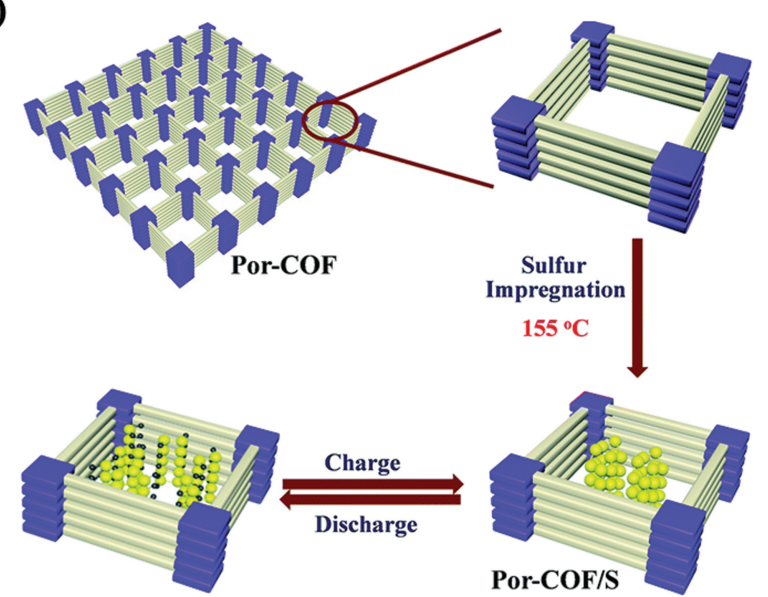

Figure 7 (a) Synthesis of Por-COF from the condensation of p-Por-CHO and PPD. (b) Schematic representation of the synthesis of the Por-COF/S composite and its charge/discharge process. Adapted with permission from Ref. 55. Copyright 2016 The Royal Society of Chemistry.

chemically reduced, forming short-chain PSs. These shortchain PSs return back to the cathode where they are reoxidized. This continuous process leads to decreased active mass utilization and leakage of active material resulting in low capacities, poor cycling, and low energy efficiency. ${ }^{57}$ Research has been devoted to tuning the nanostructures of carbon materials to limit the generation of PSs and retain the active sulfur species within the sulfur electrodes. Microporous materials that allow for the diffusion of Li-ions and facile electron transfer have proven to be advantageous. 58,59

Lithium PSs can generally be stabilized by two classes of chemical interactions, lithiophilic and sulphilic. Wang and coworkers designed a COF incorporating boroxine and triazine units to utilize both classes of chemical interactions in one structure. ${ }^{60} \mathrm{~TB}-\mathrm{COF}$ was synthesized via the self-condensation of 4-cyanophenylboronic acid (Figure 9a). By using TB-COF as a sulfur host material, Wang and coworkers hoped to investigate the amphiphilic interactions of PSs in $\mathrm{Li}-\mathrm{S}$ batteries. A melt-diffusion method was employed to prepare $\mathrm{TB}-\mathrm{COF} / \mathrm{S}$. There was a reported decrease in surface area from 708 to $2 \mathrm{~m}^{2} \mathrm{~g}^{-1}$ as well as a corresponding decrease in pore a)

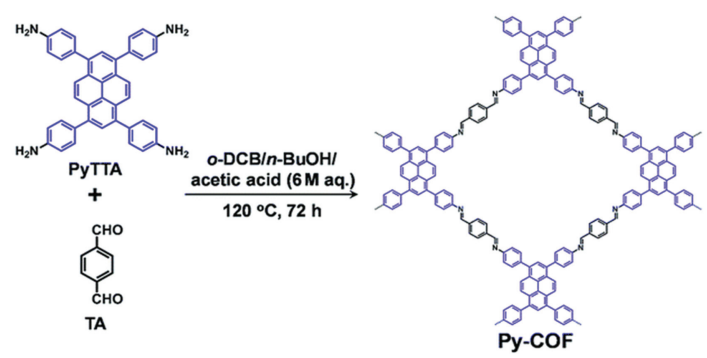

b)

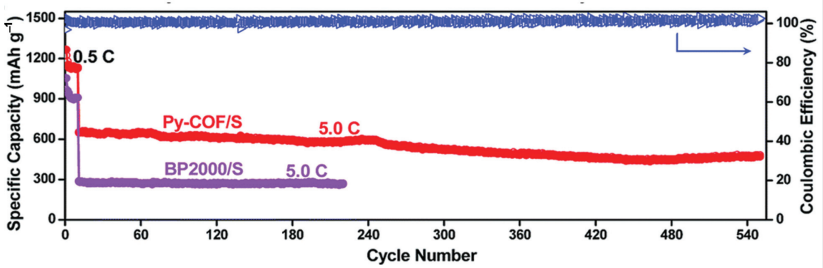

Figure 8 (a) Synthesis of Py-COF from the condensation of 1,3,6,8tetrakis(4-aminophenyl)pyrene (PyTTA) and terephthalaldehyde (TA). (b) Cycling performance of Py-COF/S and BP2000/S at 5.0 C, where the first 10 cycles correspond to an activation cycle run at $0.5 \mathrm{C}$. Adapted with permission from Ref. 56. Copyright 2018 The Royal Society of Chemistry.

volume from 0.28 to $0.04 \mathrm{~cm}^{3} \mathrm{~g}^{-1}$, indicating the successful diffusion of sulfur through the bulk material. The sulfur content of the TB-COF/S composite was determined to be $40 \mathrm{wt} \%$ by TGA. Coin cells were fabricated using the TB-COF/S composite as the cathode. The electrochemical performance of the electrode was investigated at $0.2 \mathrm{C}$. Initial cycling data show a coulombic efficiency of over $100 \%$, which is attributed to the incomplete activation of the cathode. During these initial cycles, the sulfur particles are converted into PSs, which are then effectively trapped in TB-COF due to favorable interactions along the pore wall. This allows facile diffusion of the electrolyte and Li-ions. The entrapment of the PSs in the composite allows the remaining sulfur species to rearrange in electrochemically stable sites. Nyquist plots of the composite indicate minimal leakage of sulfur into the electrolyte solution after the cathode activation process. At $0.2 \mathrm{C}$ the TB-COF/S electrode achieved a maximum capacity of $1390 \mathrm{mAhg}^{-1}$ and maintained a capacity of $945 \mathrm{~mA} \mathrm{~h} \mathrm{~g}^{-1}$ after 250 cycles with a stable coulombic efficiency, indicating the success of sulfur entrapment in the pores of the electrode (Figure 9b). Notably, the electrode still delivered high reversible capacities at 1 and 3 Cof 670 and $432 \mathrm{mAh} \mathrm{g}^{-1}$, respectively (Figure 9c). After 800 cycles, the electrode displayed reversible capacities of 663 and $482 \mathrm{~mA} \mathrm{~h} \mathrm{~g}^{-1}$ for current rates of 1 and $2 \mathrm{C}$, respectively. To investigate the impact of the introduction of lithiophilic and sulphilic groups in one material, two more COFs were synthesized, one containing only triazine rings (T-COF) and one containing only boroxine rings (B-COF). Examination of their electrochemical performances showed that TB-COF/S displayed the highest discharge and recovered capacities, 
a)

b)
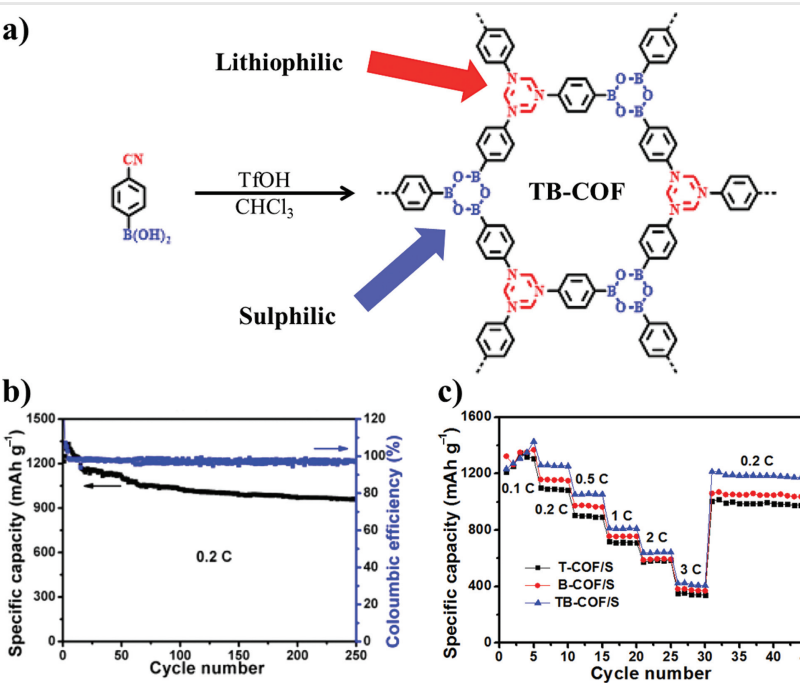

c)

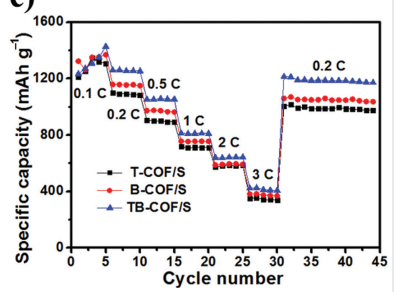

Figure 9 (a) Synthesis of TB-COF containing both lithiophilic and sulphilic sites. (b) Cycling performance of TB-COF/S at $0.2 \mathrm{C}$. (c) Capacities of T-COF/S, B-COF/S, and TB-COF/S at different current densities ranging from 0.1 to $3 \mathrm{C}$. Reprinted with permission from Ref. 60. Copyright 2018 Elsevier.

indicating the importance of the amphiphilic nature of the material. Ultimately, the cooperative effect of lithiophilic and sulphilic interactions produced an electrode that demonstrated high cycling stability and coulombic efficiency as a direct result of the interactions mitigating the dissolution of PSs into the electrolyte and free migration toward the anode.

Utilizing an inverse vulcanization process, ${ }^{61}$ Segura and coworkers designed a 2D-COF, $\mathrm{H}_{2} \mathrm{P}-\mathrm{COF}$-BATA, with accessible allyl moieties in the pores (Figure 10a). ${ }^{62}$ The inverse vulcanization process was employed to make strong $\mathrm{C}-\mathrm{S}$ bonds between sulfur and the allyl groups via radical copolymerization. The electrochemical performance of this COF was examined along with the previously reported allylfree $\mathrm{H}_{2} \mathrm{P}-\mathrm{COF}$ to examine the synergistic effect of the physical encapsulation and covalent binding of sulfur in the COF's pores. After sulfur incorporation, both COFs showed a significant decrease in surface area from 435 to $34 \mathrm{~m}^{2} \mathrm{~g}^{-1}$ for $\mathrm{H}_{2} \mathrm{P}-\mathrm{COF}-\mathrm{BATA}$ and from 490 to $38 \mathrm{~m}^{2} \mathrm{~g}^{-1}$ for $\mathrm{H}_{2} \mathrm{P}-\mathrm{COF}$. Similarly, decreases were observed in pore volume from 0.187 to $0.014 \mathrm{~cm}^{3} \mathrm{~g}^{-1}$ for $\mathrm{H}_{2} \mathrm{P}-\mathrm{COF}-\mathrm{BATA}$ and from 0.187 to $0.014 \mathrm{~cm}^{3} \mathrm{~g}^{-1}$ for $\mathrm{H}_{2} \mathrm{P}-\mathrm{COF}$. $@ \mathrm{H}_{2} \mathrm{P}-\mathrm{COF}$ displayed a coulombic efficiency approaching $90 \%$ at a current rate of $0.1 \mathrm{C}$ and a specific discharge capacity of $\sim 900 \mathrm{~mA} \mathrm{~h} \mathrm{~g}^{-1}$ after 100 cycles (Figure 10b), though it was commented that there was a wide dispersion of values most likely attributed to the shuttle effect of PS intermediates. S@ $\mathrm{H}_{2} \mathrm{P}-\mathrm{COF}-\mathrm{BATA}$ also displayed a discharge capacity of $\sim 900 \mathrm{~mA} \mathrm{~h} \mathrm{~g}^{-1}$ after 100 cycles but with a coulombic efficiency of $95 \%$ (Figure 10c). While cycling values were similar, it was found that the electrochemical stability during extended cycling was improved for $\mathrm{S}_{2} \mathrm{H}_{2} \mathrm{P}-$ COF-BATA, indicating a dramatic decrease in the shuttle effect. Rate performance was measured at increasing current loadings of $0.1,0.2$, and $0.5 \mathrm{C}$, cycled five times at each current loading (Figure 10d, e). During this process, $\mathrm{S} @ \mathrm{H}_{2} \mathrm{P}-\mathrm{COF}-\mathrm{BATA}$ slightly outperformed $\mathrm{S} @ \mathrm{H}_{2} \mathrm{P}-\mathrm{COF}$ delivering specific capacities of 1200,550 , and $190 \mathrm{~mA} \mathrm{~h}^{-1}$ in comparison to 975,520 , and $150 \mathrm{~mA} \mathrm{~h} \mathrm{~g} \mathrm{~g}^{-1}$ at the corresponding current rates, respectively. When the current loading was returned to the initial rate of $0.1 \mathrm{C}$, S@ $\mathrm{H}_{2} \mathrm{P}-\mathrm{COF}-\mathrm{BATA}$ and $\mathrm{S} @ \mathrm{H}_{2} \mathrm{P}-\mathrm{COF}$ displayed specific capacities of 1100 and $810 \mathrm{~mA} \mathrm{~h} \mathrm{~g}^{-1}$, respectively, demonstrating the improved rate capability of S@ $\mathrm{H}_{2} \mathrm{P}-\mathrm{COF}-\mathrm{BATA}$. It is suggested that the covalent linkage of sulfur intermediates inhibits sulfur release, effectively decreasing the shuttle effect, thereby increasing the electrochemical performance of the battery.

\section{Enhancing Performance via Exfoliation}

The large 1D pore channels of COFs are very beneficial for enabling high solvent permeability. ${ }^{63,64}$ However, the dense face-to-face packing of the 2D layers can restrict intercalation of ions deep into the bulk material resulting in sluggish ion kinetics. Thus, as the cell cycles, ions must migrate along relatively lengthy portions of these 1D channels, hindering the number of utilized active sites, particularly at higher current loadings. To address this issue, strategies have been developed to chemically or mechanically exfoliate COFs from their bulk form into processed materials with fewer layers.

Feng, Wang, and coworkers utilized diaminoanthraquinone (DAAQ) along with triformylphloroglucinol (TFP) to form DAAQ-TFP-COF (Figure 11a), which was then successfully delaminated mechanically via a ball-milling technique. ${ }^{65}$ This exfoliated COF, named DAAQ-ECOF, achieved a capacity of $145 \mathrm{~mA} \mathrm{~h} \mathrm{~g}^{-1}$, which correlates to $96 \%$ utilization of its active sites. It also showed two- and three-fold capacity performance enhancements compared to the nonexfoliated DAAQ-TFP-COF at 500 and $3000 \mathrm{~mA} \mathrm{~g}^{-1}$ current loadings, respectively (Figure 11c). Remarkably, $98 \%$ capacity retention was observed after 1800 cycles of deep charge/discharge cycles. The authors attributed this enhanced performance to the drastic increase in site accessibility, as evidenced by the changes in CVs for DAAQ-TFP-COF and DAAQ-ECOF. Before exfoliation, the cathodic and anodic peaks grew linearly with the square root of the scan rate, which is indicative of redoxreaction kinetics bottle-necked by Li-ion diffusion through the material. Conversely, after exfoliation, the cathodic and anodic peaks grew linearly with changes in sweep rate, indicating surface or near-surface redox processes. This suggests that the kinetics were mainly determined by charge transfer processes. In other words, the diffusion of Li-ions to active sites was essentially unhindered, and charge/discharge processes were limited only by how quickly charges could reach the active sites. This imbued the exfoliated COF with 

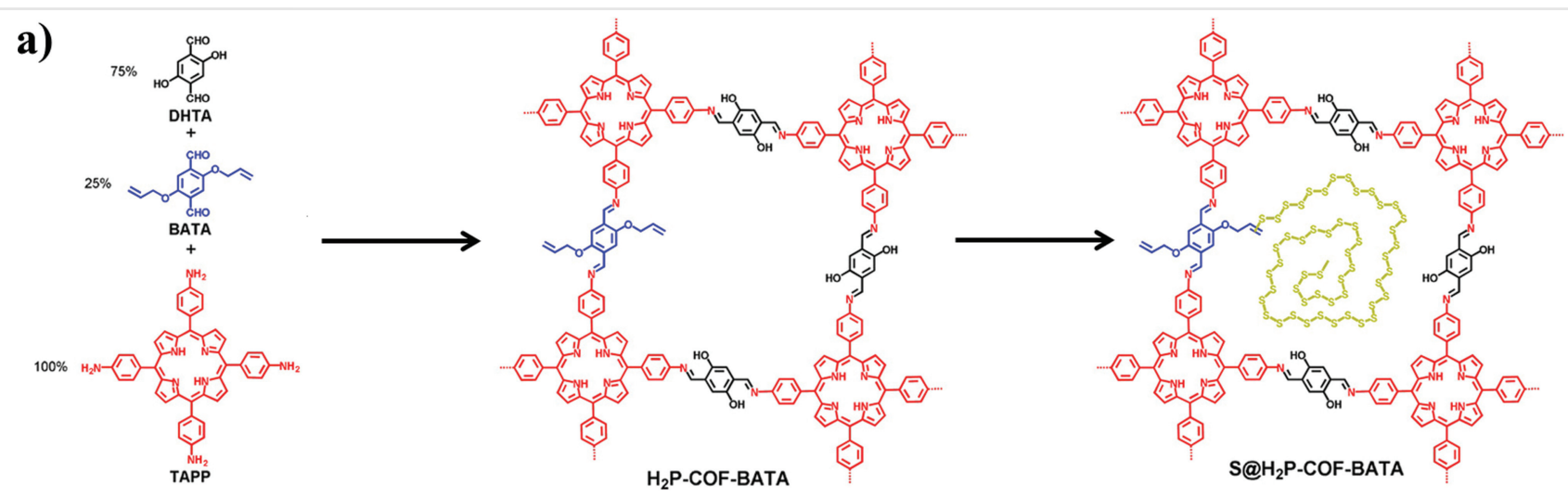

b)

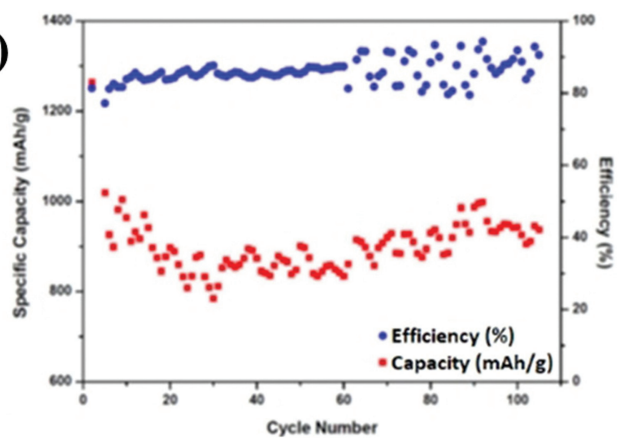

d)

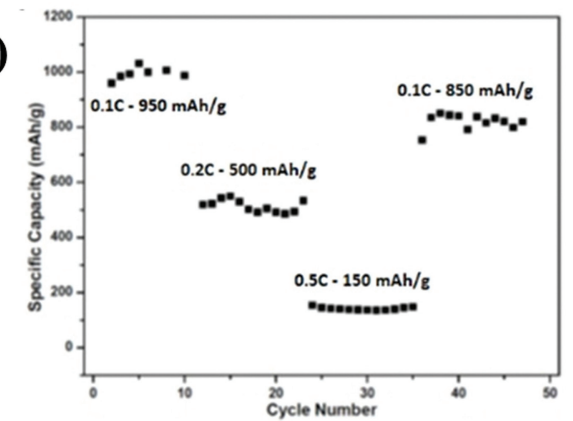

c)

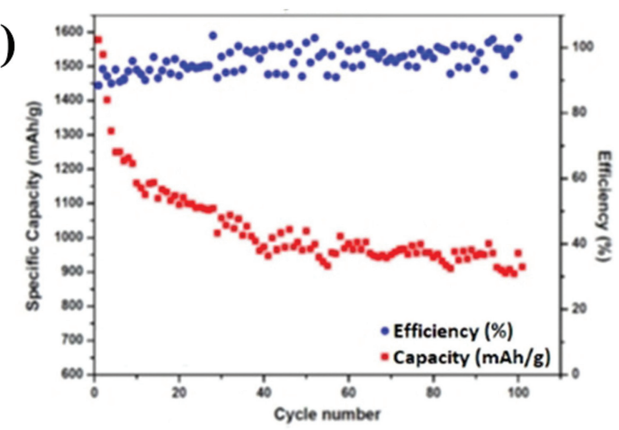

e)

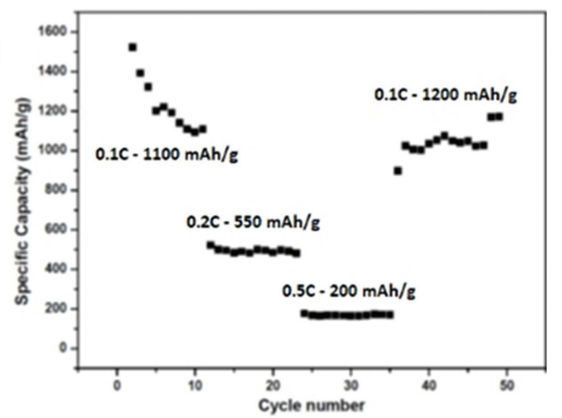

Figure 10 (a) Synthesis of $\mathrm{H}_{2} \mathrm{P}$-COF-BATA and $\mathrm{S} @ \mathrm{H}_{2} \mathrm{P}$-COF-BATA. Cycling performance of (b) $\mathrm{S}_{\mathrm{C}} \mathrm{H}_{2} \mathrm{P}$-COF and (c) S@H $\mathrm{H}_{2} \mathrm{P}$-COF-BATA at $0.1 \mathrm{C}$. Capacities of (d) $\mathrm{S}_{\mathrm{H}} \mathrm{P}$-COF and (e) $\mathrm{S}_{2} \mathrm{H}_{2} \mathrm{P}-\mathrm{COF}-\mathrm{BATA}$ at different scan rates from 0.1 to $1.0 \mathrm{C}$. Adapted with permission from Ref. 62. Copyright 2019 Wiley-VCH Verlag $\mathrm{GmbH} \& \mathrm{Co}$.

ultrafast charge/discharge performance and nearly full utilization of its active sites.

This method of performance enhancement was demonstrated to be generalizable to two other structures presented by the authors as well (Figure 11a). By using diaminobenzoquinone (DABQ) linkers instead of DAAQ DABQ-TFP-COF was synthesized. Additionally, TEMPO-COF was synthesized by combining 1,3,5-tris-(4-aminophenyl) benzene (TAPB) with 2,5-bis(2-propynyloxy)terephthalaldehyde, followed by appending an azide-modified TEMPO via a copper-mediated click reaction postsynthetically. Both COFs showed marked improvement in performance upon exfoliation (Figure 11e), and voltage profiles obtained from galvanostatic discharging demonstrate the expected trends in charge storage performance (Figure 11d). At $20 \mathrm{~mA} \mathrm{~g}^{-1}$, capacities for DABQ-ECOF and TEMPO-ECOF were determined to be $35 \%$ and $53 \%$ higher than their nonexfoliated counterparts, respectively, demonstrating the distinct advantage exfoliation can have on both the capacity and kinetics for COF-based electrode materials.

Ajayan, Chen, Wang, and coworkers developed an approach to chemically exfoliate their COF structure into few-layered covalent organic nanosheets (CONs) for significantly improved cycling. ${ }^{66}$ Schiff base condensation reactions between 1,2,4,5-tetrakis-(4-formylphenyl)benzene (TFPB) and PPD yielded TFPB-COF (Figure 12a), which could then be chemically exfoliated under strong oxidative conditions, followed by washing to remove impurities to 


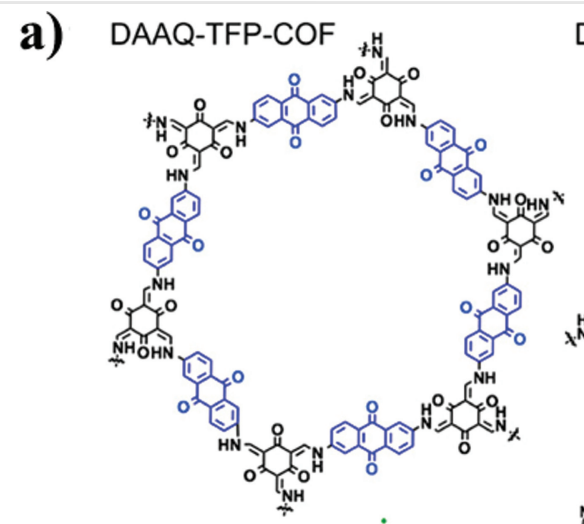

DABQ-TFP-COF

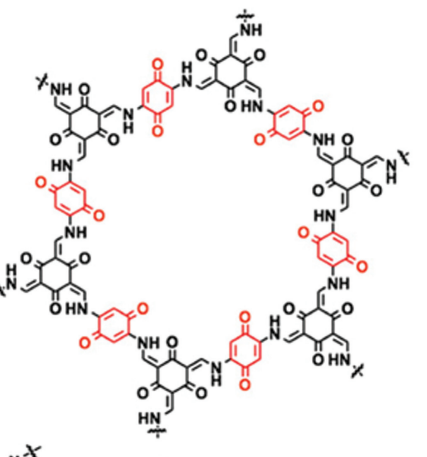

TEMPO-COF

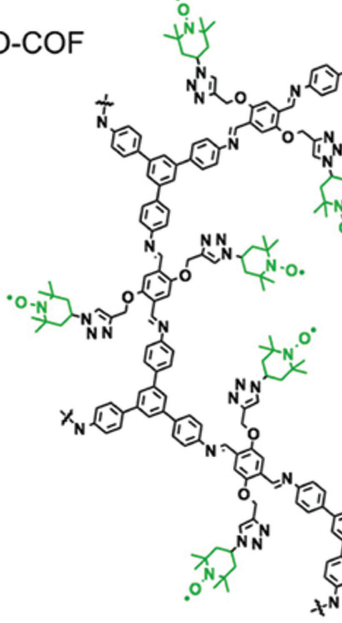

b)

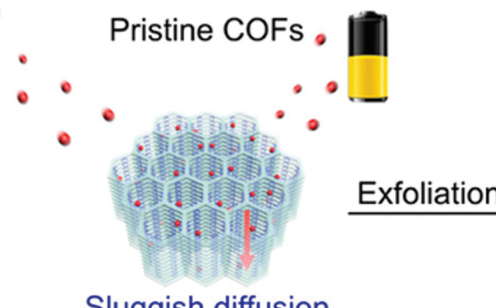

Sluggish diffusion

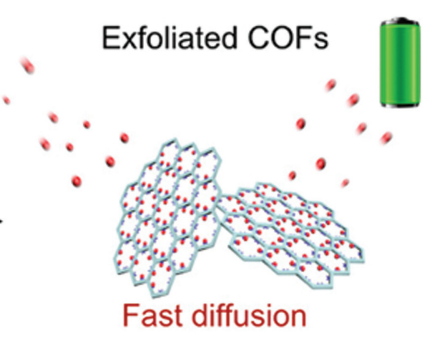

c)

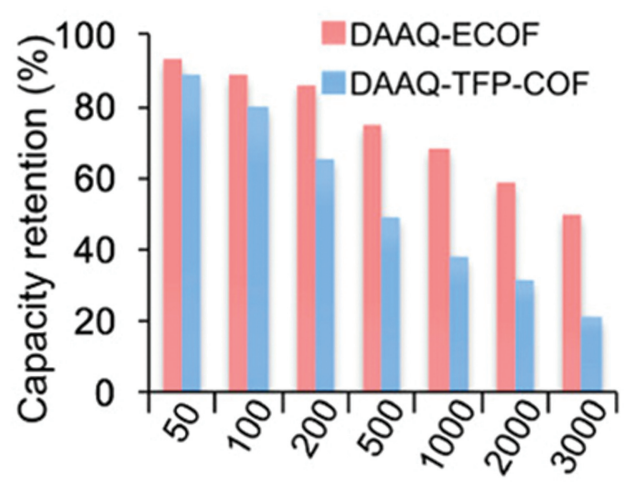

d)

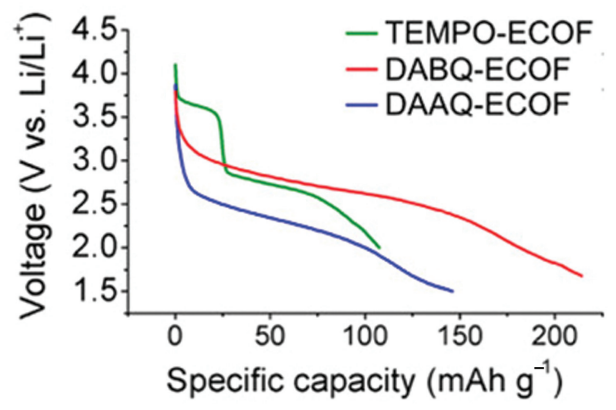

e)

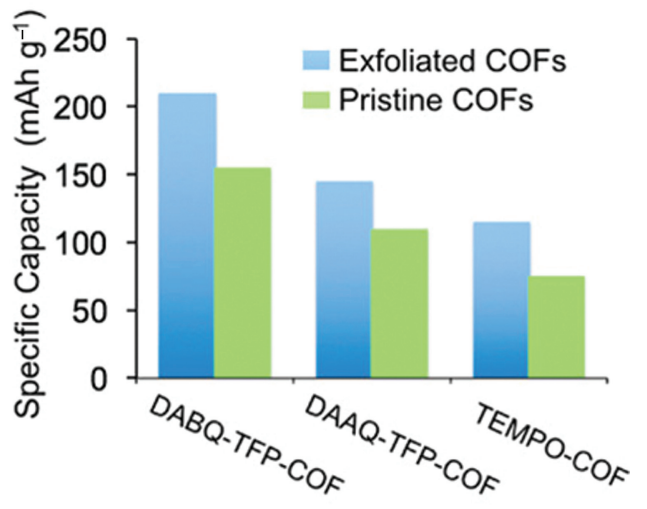

Figure 11 (a) Structures of DAAQ-TFP-COF, DABQ-TFP-COF, and TEMPO-COF. (b) Schematic representation of the exfoliation of 2D COFs into exfoliated COFs. (c) Cycling performances and coulombic efficiencies of DAAQ-ECOF and DAAQ-TFP-COF at $500 \mathrm{~mA} \mathrm{~g}^{-1}$. (d) Discharge curves for TEMPO-ECOF, DABQ-ECOF, and DAAQ-ECOF at $20 \mathrm{~mA} \mathrm{~g}^{-1}$. (e) Comparison of capacities of pristine and exfoliated COFs at $20 \mathrm{~mA} \mathrm{~g}^{-1}$. Adapted with permission from Ref. 65. Copyright 2017 American Chemical Society.

yield E-TFPB-COF. The successful exfoliation was confirmed by high-resolution transmission electron microscopy (HR-TEM) and XPS. After 300 cycles at $100 \mathrm{~mA} \mathrm{~g}^{-1}$ current loading, the pristine TFPB-COF produced a reversible capacity of $126 \mathrm{~mA} \mathrm{~h} \mathrm{~g}^{-1}$. In contrast, under the same conditions, E-TFPB-COF produced a reversible capacity of $968 \mathrm{~mA} \mathrm{~h} \mathrm{~g}^{-1}$ (Figure 12b). In situ Raman spectroscopy of the anode during battery cycling provided insight into the lithiation/delithiation mechanism. In the case of E-TFPBCOF, it was observed that additional redox reactions took place using the conjugated $\pi$-electrons from aromatic benzene rings which manifested in its large reversible capacities.

Vaidhyanathan, Ogale, and coworkers combined TFP and 3,5-diaminotriazole to synthesize a flexible triazole-containing COF which spontaneously self-exfoliated into CONs during synthesis (Figure 13a). ${ }^{67}$ In its calculated lowest energy state, the triazole rings acted as a pivot point, buckling to form a wavy 2D structure which was easily disrupted, causing the self-exfoliation phenomenon. During cycling, IISERP-CON1 exhibited stable cycling behavior over 100 charge/discharge cycles at $100 \mathrm{~mA} \mathrm{~g}^{-1}$ current loading, producing a reversible 
a)

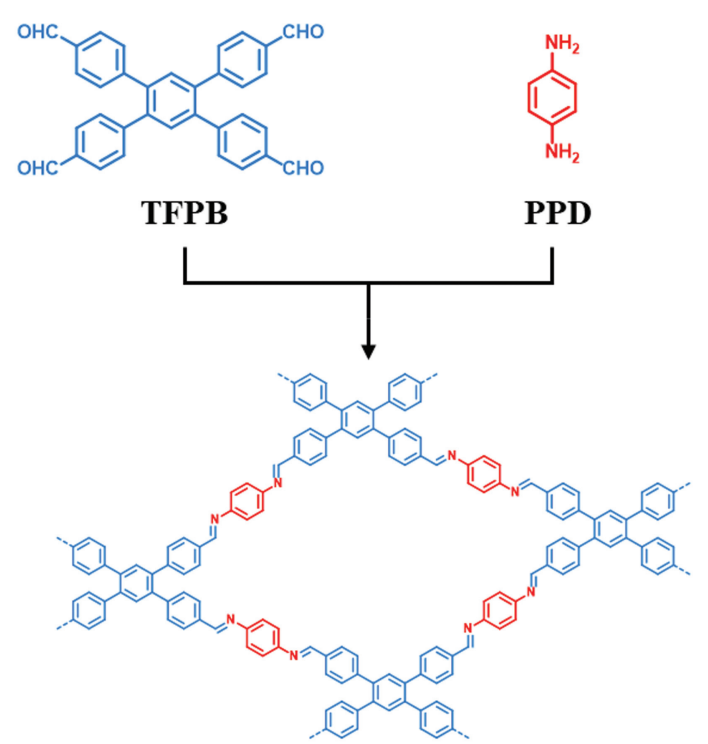

b)

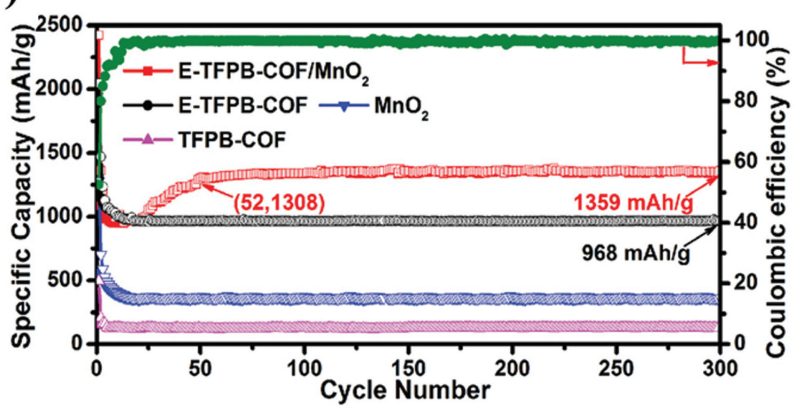

Figure 12 (a) Synthesis of TFPB-COF. (b) Cycling performances of E-TFPB-COF/ $\mathrm{MnO}_{2}$, E-TFPB-COF, TFPB-COF and commercial $\mathrm{MnO}_{2}$ at $0.1 \mathrm{~A} \mathrm{~g}^{-1}$. Adapted with permission from Ref. 66. Copyright 2019 Wiley-VCH Verlag GmbH \& Co.

capacity of $720 \mathrm{~mA} \mathrm{~h} \mathrm{~g}{ }^{-1}$ (Figure 13b). Interestingly, there was only a decrease of $150 \mathrm{~mA} \mathrm{~h} \mathrm{~g} \mathrm{~g}^{-1}$ observed when the current density was increased to $1 \mathrm{~A} \mathrm{~g}^{-1}$. IISERP-CON1 also demonstrated remarkably high coulombic efficiency of almost $100 \%$ throughout cycling. The authors further probed the cycling stability by cycling cells at $500 \mathrm{~mA} \mathrm{~g}^{-1}$. At the 1000th cycle, $98 \%$ capacity retention was observed, along with no significant change indicated in the resistance of the cell, as determined by electrochemical impedance spectroscopy (EIS). Additionally, cycling of the neat material over 100 charge/discharge cycles showed no change in the crystal or chemical structure. These results indicate a lack of ohmic loss or material degradation throughout the entire cycling process.

Huang, Zhao, and coworkers utilized mechanical exfoliation to improve the performance of their COF system compared to the bulk material. ${ }^{68}$ Combination of pyromellitic dianhydride (PMDA) with 1,3,5-tris(4-aminophenyl)amine

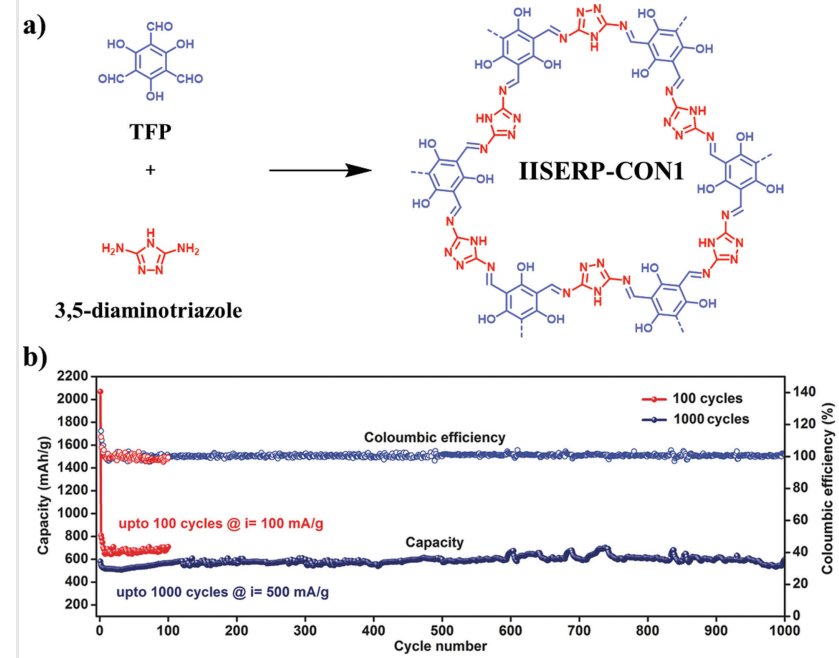

Figure 13 (a) Synthesis of IISERP-CON1. (b) Cycling performance at $100 \mathrm{~mA} \mathrm{~g}^{-1}$ (red, up to 100 cycles) and $500 \mathrm{~mA} \mathrm{~g}^{-1}$ (blue, up to 1000 cycles). Adapted with permission from Ref. 67. Copyright 2018 WileyVCH Verlag $\mathrm{GmbH} \& \mathrm{Co}$.

(TAPA) or TAPB under solvothermal conditions yielded PICOF-1 and PI-COF-2, respectively (Figure 14a). These COFs were then shown to be capable of exfoliation via ball milling to produce PI-ECOF-1 and PI-ECOF-2. When utilized as cathode materials, PI-COF-1 demonstrated a reversible capacity of $85 \mathrm{mAh} \mathrm{g}^{-1}$, corresponding to $60 \%$ site utilization, at a current loading of $0.1 \mathrm{C}$. This was improved to $112 \mathrm{~mA} \mathrm{~h} \mathrm{~g} \mathrm{~g}^{-1}$, corresponding to $79 \%$ active site utilization, upon exfoliation to PI-ECOF-1. The improved performance after exfoliation is attributed in part to a more efficient transportation of $\mathrm{Li}$-ions within the cell, as signaled by the high peak current seen during CV studies compared to the nonexfoliated variant (Figure 14b). This is further indicated by the decreased value of $R_{\mathrm{ct}}$ in the Nyquist plot for PI-ECOF-1, which indicates an improved rate of charge transfer during cycling (Figure 14c).

The authors noted that during cycling, rapid capacity fading and poor coulombic efficiencies could be observed even at low current loadings, indicating the presence of parasitic side reactions which were proposed to be taking place between the carbonate-containing electrolytes and carbonyl moieties of the COF materials. Changing the Li-ion source from $\mathrm{LiPF}_{6}$ to LiTFSI, a more ionic lithium source, and from carbonate-based electrolyte to a dioxolane/dimethylether (DOL/DME) electrolyte improved cycle stability and efficiency dramatically, allowing for nearly $100 \%$ efficiency maintained over the majority of 300 cycles.

\section{Composite Materials with Carbon Nanotubes}

The process of forming hybrid materials using COFs has been shown to assist in various applications from catalysis ${ }^{69}$ to 
a)

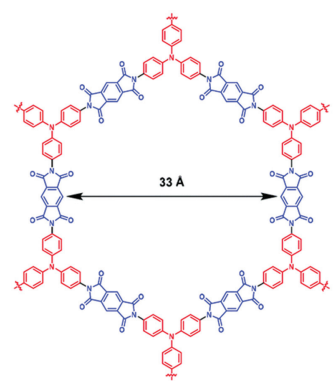

PI-COF-1 theoretical capacity: $142 \mathrm{~mA} \mathrm{~h} \mathrm{~g}^{-1}$

b)

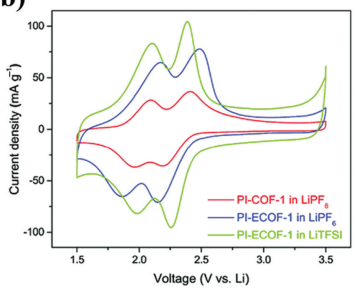

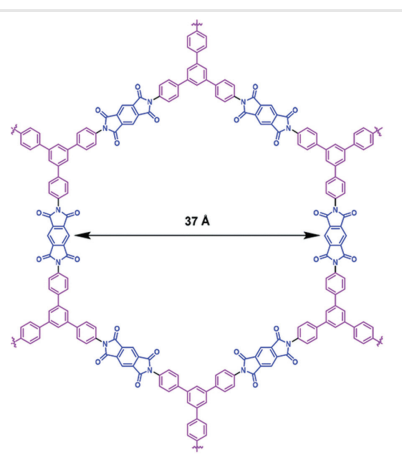

PI-COF-2 theoretical capacity: $128 \mathrm{~mA} \mathrm{~h}^{-1}$ c)

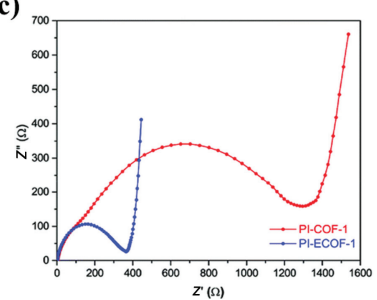

Figure 14 (a) Structures of PI-COF-1 and PI-COF-2. (b) CV curves of PICOF-1 and PI-ECOF-1 in LiPF 6 and LiTFSI at $0.1 \mathrm{mV} \mathrm{s}^{-1}$. (c) Nyquist plot of PI-COF-1 and PI-ECOF-1. Adapted with permission from Ref. 68. Copyright 2019 The Royal Society of Chemistry.

gas storage $e^{70}$ to dye separation. Often, COF-based composites are designed by one of two methods: covalent tethering or template-assisted growth. In the case of covalent tethering, prefunctionalized scaffolds serve as both covalent anchoring points as well as nucleation sites for growing COF crystallites orthogonal to the template's surface. Alternatively, the $\pi$ electron-rich COF structures can be grown directly on the scaffold's surface via $\pi-\pi$ stacking interactions, as in the case of template-assisted growth.

Feng and coworkers developed a polyarylimide-based COF@CNT composite via polycondensation of TAPA and 1,4,5,8-naphthalenetetracarboxylic dianhydride alone or in the presence of CNTs to achieve 2D-PAI and 2D-PAI@CNT, respectively (Figure 15a). ${ }^{71}$ Both materials were tested as cathodes to determine the effects of CNTs on LIB electrode performance. In its bulk form, 2D-PAI performed modestly, demonstrating a reversible capacity of $28.5 \mathrm{~mA} \mathrm{~h} \mathrm{~g}^{-1}$ when cycled at $0.1 \mathrm{C}$, corresponding to $22.6 \%$ utilization of its active sites. In contrast, using 2D-PAI@CNT improved activesite utilization to $82.9 \%$, thus enhancing the reversible capacity to $104.4 \mathrm{~mA} \mathrm{~h} \mathrm{~g}^{-1}$ (Figure $15 \mathrm{~b}$ ). It is important to note that this improvement is superior to the $65.6 \mathrm{~mA} \mathrm{~h} \mathrm{~g}^{-1}$ observed when simply mixing bulk COF and CNTs in a 1:1 mass ratio during cell fabrication, which points to a synergistic effect observed in the composite material.

During cycling, the authors noted the interesting longterm cycling stability of the bulk COF material. Over 50 cycles, $2 \mathrm{D}$-PAI retained $100 \%$ of its capacity in contrast to a

boronate ester-linked analog previously reported, which they ascribed to the improved chemical stability of imides. Remarkably, at $500 \mathrm{~mA} \mathrm{~g}^{-1}$, 2D-PAI@CNT was able to retain $100 \%$ of its cycling capacity over 8000 cycles (Figure $15 d$ ). To determine the significance of the imide structure employed, the authors also synthesized a COF@CNT composite using TAPA and PMDA to make PI-PMDA@CNT, which showed a $20 \%$ capacity loss after 50 cycles (Figure 15c). The authors attribute this loss to the difference in lithiation states between the two structures during cycling.

Because the lithium enolates from PI-PMDA are a part of five-membered rings, they show weaker resonance degree and less aromaticity than the six-membered conjugated lithium enolates from 2D-PAI. This leads to intramolecular strain and manifests as poorer chemical stability.

Jiang and coworkers also employed naphthalene units as redox-active building blocks for COF-based composites. Combining 2,3,6,7,10,11-hexahydroxytriphenylene (TP) and $N, N^{\prime}$-di-(4-boronophenyl)-naphthalene-1,4,5,8-tetracarboxylic acid diimide (NDI) yielded highly crystalline $\mathrm{D}_{\mathrm{TP}}-\mathrm{A}_{\mathrm{NDI}}$-COF (Figure 16a). ${ }^{72}$ As a cathode material, bulk $\mathrm{D}_{\mathrm{TP}}-\mathrm{A}_{\mathrm{NDI}}-\mathrm{COF}$ performed modestly, producing a reversible capacity of $21 \mathrm{~mA} \mathrm{~h} \mathrm{~g}^{-1}$ after 40 cycles at $200 \mathrm{~mA} \mathrm{~g}^{-1}$, corresponding to only a $26 \%$ utilization of its redox-active imide units. EIS determined that this result may be due to high levels of electrical resistance within the material in the amount of $129 \Omega$. Synthesizing the CNT composite material, $\mathrm{D}_{\mathrm{TP}}-\mathrm{A}_{\mathrm{NDI}}$-COF@CNT, drastically decreased the resistivity of the material to only $8.5 \Omega$ (Figure $16 \mathrm{~b}$ ). This translated into a dramatically increased utilization of active sites to $82 \%$ during cycling, corresponding to a reversible capacity of $67 \mathrm{~mA} \mathrm{~h} \mathrm{~g}^{-1}$ at $200 \mathrm{~mA} \mathrm{~g}^{-1}$ (Figure 16c). Notably, the coulombic efficiency remained close to $100 \%$ during cycling, even when current loadings were increased as high as $12 \mathrm{C}$ and charging/discharging was continued to 700 cycles, demonstrating the excellent high-rate performance and long-term stability of the material in comparison to conventional organic cathodes.

\section{Composite Materials with Graphene}

In addition to the exfoliation of PI-COF-1 and PI-COF-2 discussed earlier in this review, Huang, Zhao, and coworkers utilized the $\pi$-electron-rich structure and perceived advantages of reduced graphene oxide's (rGO) high conductivity to design a COF-based graphene composite for use as a LIB cathode material. ${ }^{68}$ Preparation via ball milling produced PI-COF-1/rGO $x$, where $x$ describes the wt\% loading of $\mathrm{rGO}$ during synthesis. Increasing from 0 to 10,30 , and then $50 \%$ loadings improved capacity from 103 to 110, 127, and $167 \mathrm{~mA} \mathrm{~h} \mathrm{~g}^{-1}$, respectively, when cycled at $0.1 \mathrm{C}$ (Figure 17a). Subtracting out the contribution of the rGO substrate allowed calculation of the total active-site utilization 
a)

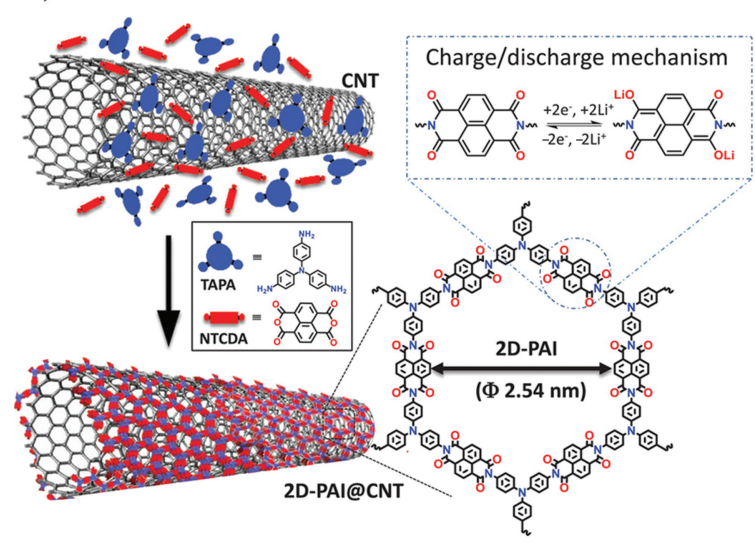

b)

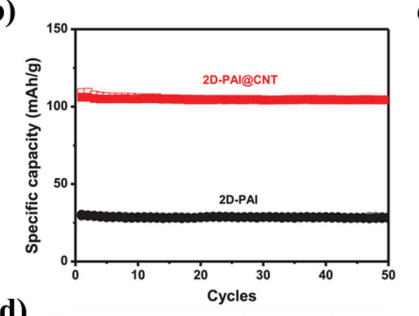

d)

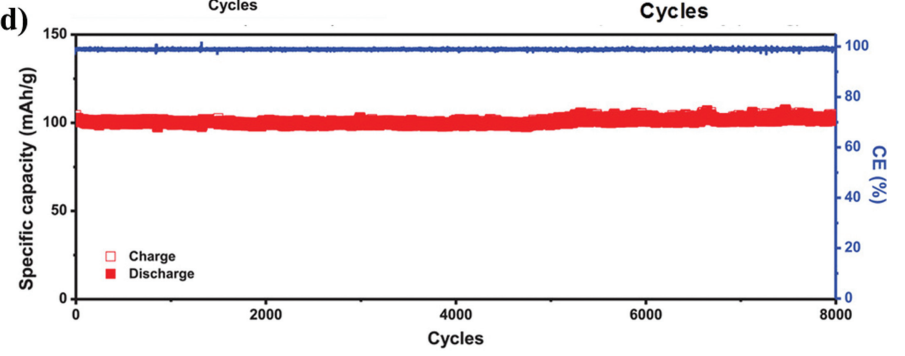

c)

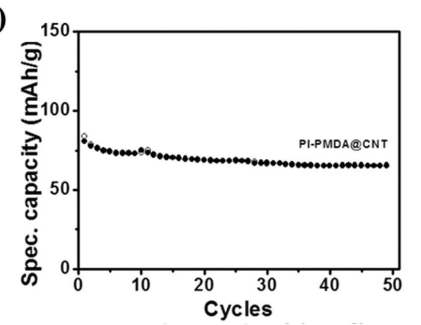

Cycles

Figure 15 (a) Schematic representation of the synthesis of 2D-PAI@CNT and its energy-storage process. (b) Cycling performance of 2D-PAI and 2D-PAI@CNT at $0.1 \mathrm{~A} \mathrm{~g}^{-1}$. (c) Cycling performance of PI-PMDA@CNT at $100 \mathrm{~mA} \mathrm{~g}^{-1}$. (d) Long-term cycling stability of 2D-PAI@CNT at $0.5 \mathrm{~A} \mathrm{~g}{ }^{-1}$. Adapted with permission from Ref. 71. Copyright 2019 Wiley-VCH Verlag GmbH \& Co.

a)

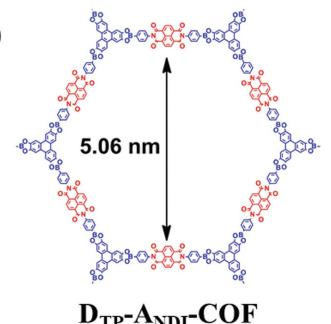

c)
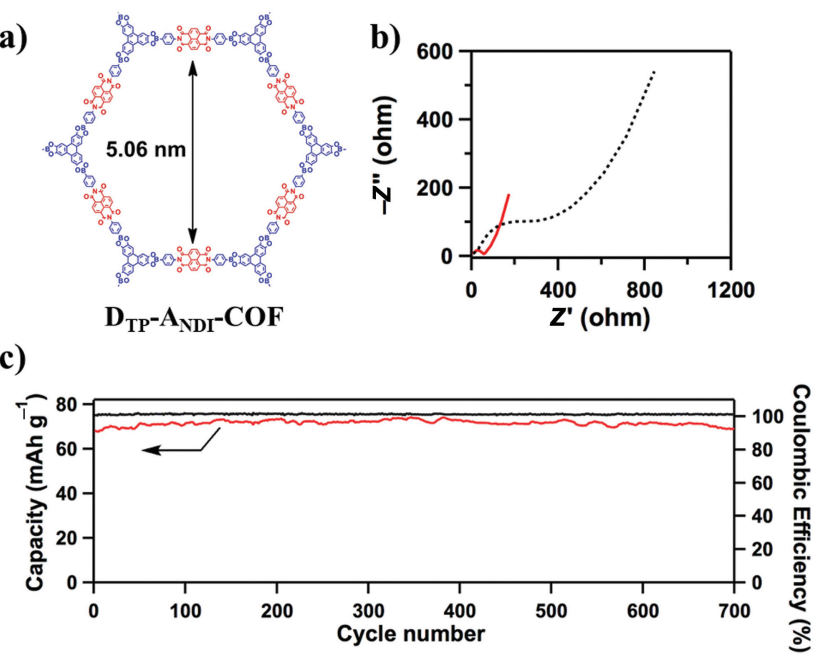

Figure 16 (a) Structure of $D_{T P}-A_{N D I^{-}} C O F$. (b) Nyquist plot of $D_{T P}-A_{N D I^{-}}$ COF (dotted black curve) and $\mathrm{D}_{\mathrm{TP}}-\mathrm{A}_{\mathrm{NDI}}$-COF@CNTs (red curve). (c) Capacity (red line) and coulombic efficiency (black line) of $\mathrm{D}_{\mathrm{TP}^{\mathrm{P}}}-\mathrm{A}_{\mathrm{NDI}}$ COF@CNTs at 2.4 C. Adapted with permission from Ref. 72. Copyright 2015 Springer Nature.

in each material. With no rGO, only $72 \%$ of active sites were utilized. Increasing to 10,25 , and $50 \%$ rGO loadings improved active-site utilization to 74,79 , and $\sim 100 \%$, respectively. This performance proved to be stable at coulombic efficiencies of $\sim 100 \%$ over 300 cycles at current loadings of $0.1 \mathrm{C}$ (Figure 17b). Remarkably, while PI-ECOF1 shows no electrochemical activity at a current loading of $10 \mathrm{C}, \mathrm{PI}-\mathrm{ECOF}-1 / \mathrm{rGO} 30$ and PI-ECOF-1/rGO50 produced capacities of 60 and $90 \mathrm{~mA} \mathrm{~h} \mathrm{~g}^{-1}$, corresponding to $60 \%$ and $67 \%$ of their total theoretical capacities.
Similarly, performance enhancement was observed between the exfoliated PI-ECOF- 2 and the PI-ECOF-2/rGO30 composite material. Before the addition of graphene, PI-ECOF-2 achieved a reversible capacity of $103 \mathrm{~mA} \mathrm{~h} \mathrm{~g}^{-1}$ at a cycling rate of $0.1 \mathrm{C}$, corresponding to utilization of $80 \%$ of its active sites. However, when tested as the composite material, PI-ECOF-2/ rGO30 showed an enhanced capacity of $128 \mathrm{~mA} \mathrm{~h} \mathrm{~g}{ }^{-1}$, corresponding to $97 \%$ utilization of its active sites (Figure 17c). In addition, cycling at higher rates showed improvement when in a composite material. Reversible capacity when charging/discharging at $5 \mathrm{C}$ increased from $10 \mathrm{~mA} \mathrm{~h} \mathrm{~g}{ }^{-1}$ with PI-ECOF-2 to $37 \mathrm{~mA} \mathrm{~h} \mathrm{~g}{ }^{-1}$. Notably, the performance proved very stable, maintaining consistent capacity values and nearly $100 \%$ coulombic efficiency over 300 cycles (Figure 17d). The authors note that this demonstrates the significant impact graphene-based composite materials seem to have in energy-storage applications.

\section{Composite Materials with Nanoparticles}

As was mentioned previously in this review, Ajayan, Chen, Wang, and coworkers developed an imine-linked COF, TFPBCOF, which was amenable to improved LIB anode performance via chemical stripping exfoliation. ${ }^{66}$ The method of chemical stripping involved the use of highly oxidizing conditions which resulted in the production of $\mathrm{MnO}_{2}$ nanoparticles (NPs), the presence of which served to prevent the reaggregation of the sheets back into a bulk structure via physically limiting their contact distances (Figure 18a). While the NPs could be washed away to afford purified E-TFPB-COF, the authors also isolated E-TFPB-COF/ $\mathrm{MnO}_{2}$ for testing as a composite anode material for LIBs. Cycling E-TFPB-COF and 

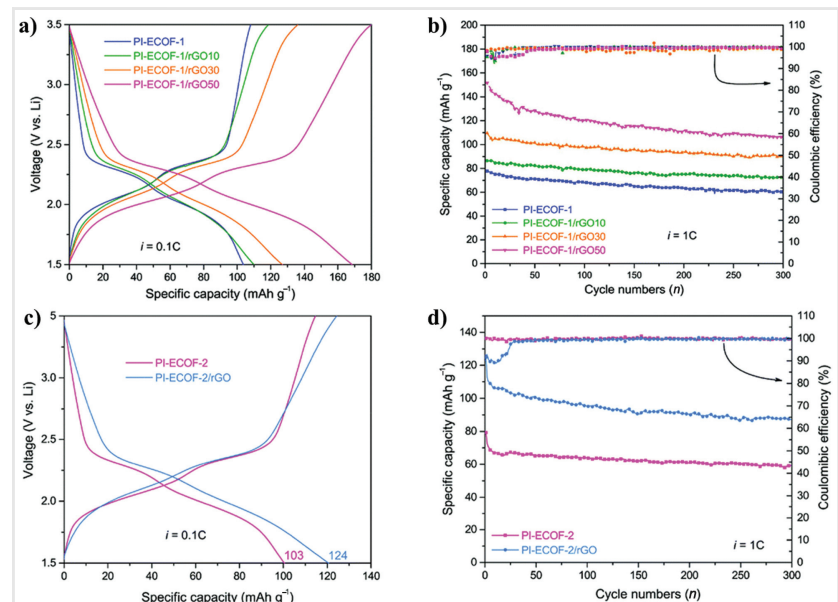

Figure 17 (a) Charge/discharge profiles of the second cycle at $0.1 \mathrm{C}$ of $\mathrm{PI}$-ECOF-1 and its rGOX composites. (b) Coulombic efficiency and cycling stability at $1 \mathrm{C}$ of PI-ECOF- 1 and its rGOX composites. (c) Charge/discharge profile of the second cycle at $0.1 \mathrm{C}$ of PI-ECOF- 2 and $\mathrm{PI}-\mathrm{ECOF} / \mathrm{rGO}$. (d) Coulombic efficiency and cycling stability at $1 \mathrm{C}$ of PIECOF-2 and PI-ECOF/rGO. Adapted with permission from Ref. 68. Copyright 2019 The Royal Society of Chemistry.

E-TFPB-COF/ $/ \mathrm{MnO}_{2}$ for 300 cycles demonstrated extremely high reversible capacity values of 968 and $1359 \mathrm{~mA} \mathrm{~h} \mathrm{~g}^{-1}$, respectively, both of which significantly outperform the pristine bulk material's capacity of $126 \mathrm{~mA} \mathrm{~h} \mathrm{~g}^{-1}$. The composite material also proved tolerant to harsh redox conditions, including charge/discharge rates as high as $5 \mathrm{Ag}^{-1}$ (Figure 18b).

Lou, $\mathrm{Ci}$, and coworkers utilized Si NPs as a template for COF synthesis with the intention of providing a robust, artificial solid-electrolyte interphase (SEI) layer. $^{73}$ Combining $\mathrm{Tp}$ with 2,5-diaminobenzenesulfonic acid and prefabricated $\mathrm{Si}$ NPs under solvothermal conditions yielded Si@COF NPs (Figure 19a). When assembled into a LIB, Si@COF demonstrated stability and efficiency that was markedly improved over uncoated Si NPs. Introduction of the COF structure slightly decreased the reversible capacity from 2869 to $2761 \mathrm{~mA} \mathrm{~h} \mathrm{~g}^{-1}$ at a cycling rate of $500 \mathrm{~mA} \mathrm{~g}^{-1}$. However, the coulombic efficiency for Si@COF during the initial charge/discharge cycle increased from $\sim 71 \%$ with pristine $\mathrm{Si}$ NPs to 82\% with Si@COF, which the authors ascribe to the disfavoring of SEI growth in the presence of the artificial COFbased SEI layer. This is further corroborated by the absence of peaks correlated to SEI growth in CVs taken of both the composite materials (Figure 19c, d). Additionally, the material showed dramatically improved cycling performance at extremely high current loadings. When cycled at $5000 \mathrm{~mA} \mathrm{~g}^{-1}$, pristine Si NPs produced a reversible capacity of $371 \mathrm{~mA} \mathrm{~h} \mathrm{~g}^{-1}$. In contrast, Si@COF demonstrated a reversible capacity of $962 \mathrm{~mA} \mathrm{~h} \mathrm{~g}^{-1}$ (Figure 19b). Cross-sections of both materials were measured to determine volume expansion as a result of a)

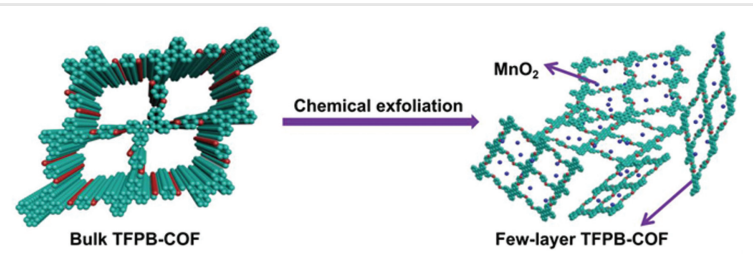

b)

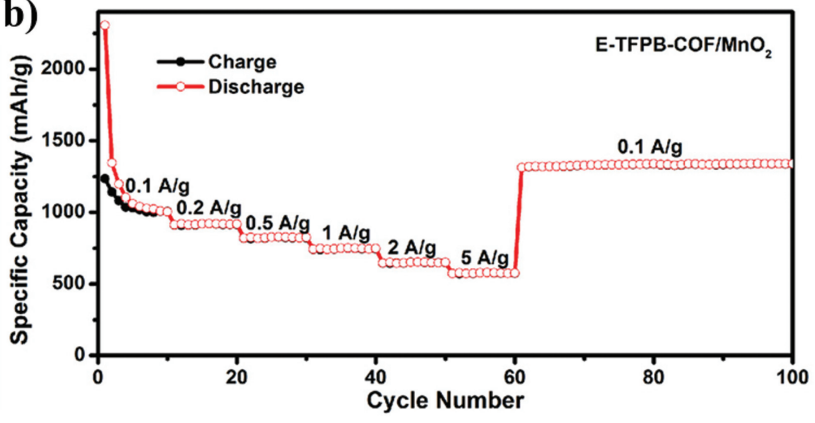

Figure 18 (a) Schematic representation of the chemical exfoliation of TFPB-COF to E-TFPB-COF/MnO 2 . (b) Rate performance of E-TFPB-COF/MnO at different current densities ranging from 0.1 to $5 \mathrm{~A} \mathrm{~g}^{-1}$. Adapted with permission from Ref. 66. Copyright 2019 Wiley-VCH Verlag GmbH \& Co.

SEI formation, which is a common source of poor cycle life in Sibased electrode materials (Figure 19e). Expansion of the Si@COF material was $80 \%$ on average, compared to $160 \%$ expansion for noncomposite materials, signaling that the artificial SEI disfavors additional growth of SEI via electrolyte decomposition. The authors note that preventing SEI formation should also provide significant long-term cycling stability. Cycling lifetimes were investigated at a current loading of $2000 \mathrm{~mA} \mathrm{~g}^{-1}$, wherein it was determined that $60 \%$ capacity was retained over 1000 cycles, most of which cycled at a coulombic efficiency of $\sim 100 \%$. This is remarkable improvement over pristine $\mathrm{Si}$, which lost $>50 \%$ of its capacity when cycled for 100 cycles at only $1000 \mathrm{~mA} \mathrm{~g}^{-1}$.

\section{Composite Materials with Ni-Rich Layered Oxides}

Choi and coworkers employed a pyrazine-linked COF grown on the surface of a Ni-rich $\mathrm{Ni}_{0.8} \mathrm{Co}_{0.1} \mathrm{Mn}_{0.1} \mathrm{O}_{2}$ (NCM811) cathode material (Figure 20a). ${ }^{74}$ The authors believed this composite material would protect the surface from cracking as a result of volume expansion, while simultaneously preventing cation swapping with Li-ions and the subsequent dissolution of the cathode material into the electrolyte. Combination of hexaketocyclohexane and benzenetetramine yielded Pyr-2D. When synthesized in the presence of NCM811, NCM811-Pyr-2D was obtained. During galvanostatic charge/discharge cycling, the composite material demonstrated exceptionally high coulombic 
a)

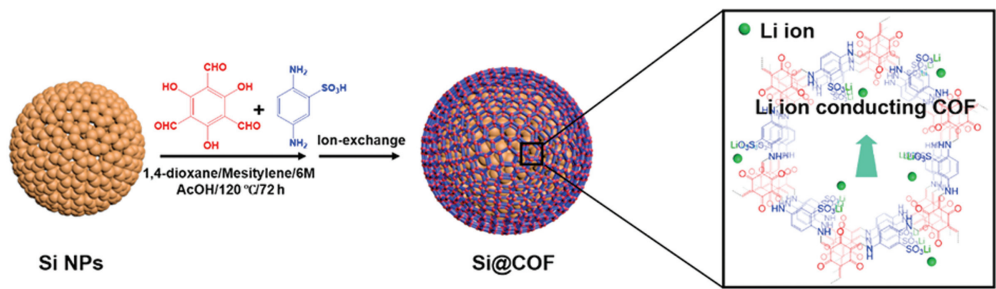

c)

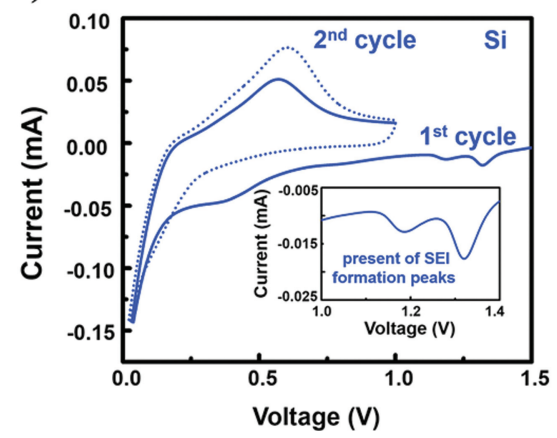

d)

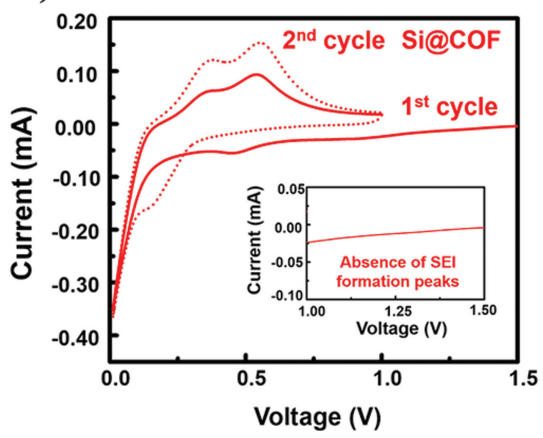

b)

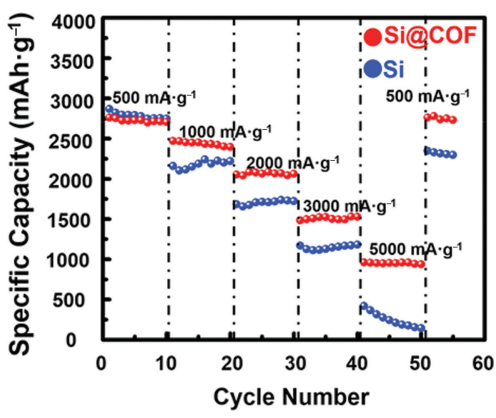

e)
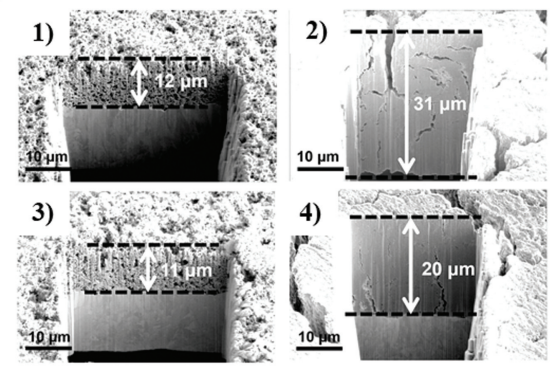

Figure 19 (a) Schematic representation of the synthesis of Si@COF NPs. (b) Rate performance of pristine Si and Si@COF. (c) CV curve of pristine Si at $0.1 \mathrm{mV} \mathrm{s}^{-1}$. (d) CV curve of Si@COF at $0.1 \mathrm{mV} \mathrm{s}^{-1}$. Cross-section SEM images of (e1) original pristine Si electrode, (e2) pristine Si electrode after 100 cycles at $1000 \mathrm{~mA} \mathrm{~g}^{-1}$, (e3) original Si@COF electrode, (e4) Si@COF electrode after 100 cycles at $1000 \mathrm{~mA} \mathrm{~g}$. Adapted with permission from Ref. 73. Copyright 2020 Elsevier.

a)

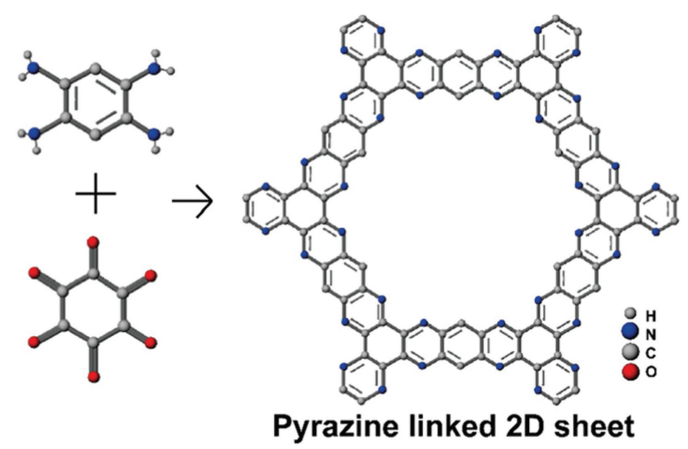

Pyrazine linked 2D sheet

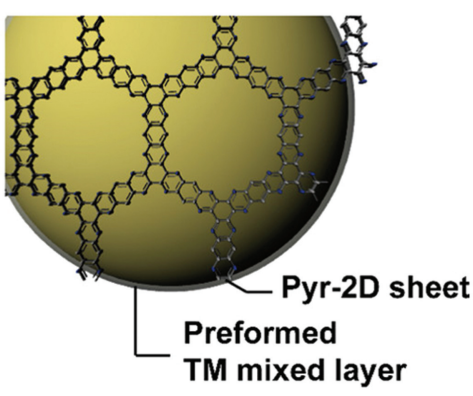

Pyr-2D sheet coated NCM811 b)

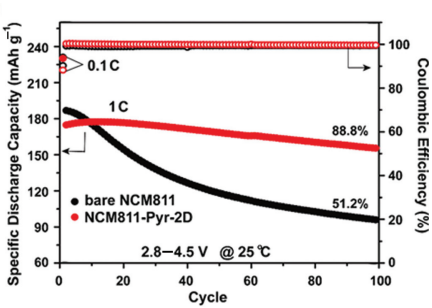

c)

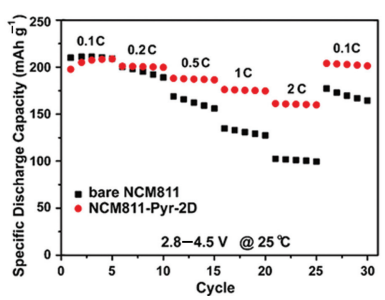

Figure 20 (a) Schematic representation of the synthesis of Pyr-2D and graphical illustration of NCM811-Pyr-2D. (b) Cycling and (c) rate performance of bare NCM811 (black) and NCM811-Pyr-2D (red). Adapted with permission from Ref. 74. Copyright 2020 American Chemical Society.

efficiency compared to the pristine material. Additionally, the NCM811-Pyr-2D electrode retained $88.8 \%$ of its original capacity after 100 cycles, which was a marked improvement compared to the $51.2 \%$ retention seen with pristine NCM811 after the same number of cycles (Figure 20b). Rate- performance studies of both materials demonstrated the superior cycling and rate performance of the NCM811-Pyr2D electrode. Whereas NCM811-Pyr-2D produced reversible capacities of $161.2 \mathrm{~mA} \mathrm{~h} \mathrm{~g}^{-1}$ when cycled at a rate of $2 \mathrm{C}$, the pristine NCM811 material was only able to produce 
a capacity of $102.3 \mathrm{~mA} \mathrm{~h} \mathrm{~g}{ }^{-1}$ (Figure 20c). The authors attributed this difference in performance to the protective role of the Pyr-2D and its ability to inhibit electrode dissolution and parasitic side reactions with the electrolyte, which are normally accelerated at high current loadings.

\section{Applications beyond Li-Ion Batteries}

While the development of Li-ion technology remains at the forefront of research, recently there has been a growing interest in utilizing other ion systems for high-performance energy-storage applications. In large part, this is due to the low abundance of lithium within the earth's crust, which ultimately limits the long-term sustainability of Li-ion batteries as an energy-storage solution. As a result, research into other more abundant ion sources such as sodium and potassium has garnered significant interest.

One common issue for batteries employing larger ions is the significant volume expansion observed during intercalation, which can be detrimental to the material's integrity. Lei and coworkers found that electrodes composed of $2 \mathrm{D}$ layered organic materials showed only a minimal volume change, making materials such as COFs a promising area to explore. $^{75}$ To investigate this in practice, Pradhan and coworkers synthesized COF TFPB-TAPT from TFPB and 1,3,5tris(4-aminophenyl)triazine (TAPT) to utilize as an anode material in a Na-ion battery (SIB) (Figure 21a). ${ }^{76}$ After evaluating the electrochemical performance at a current density of $30 \mathrm{~mA} \mathrm{~g}^{-1}$, TFPB-TAPT exhibited a capacity of $125 \mathrm{~mA} \mathrm{~h} \mathrm{~g}^{-1}$ after 500 cycles with an initial reversible capacity of $246 \mathrm{~mA} \mathrm{~h} \mathrm{~g}^{-1}$ (Figure 21b). Ex situ HR-TEM experiments were performed on the electrode at different potentials to investigate the sodium-storage mechanism and structural changes that may be taking place during the charge/discharge process. While discharging, the microstructure shows ripple-like features as a consequence of the large Na-ions inserting into the COF. The layered structure of the $\mathrm{COF}$ is restored during the charging process as the ions are removed, indicating that the nanoporous COF allows for the reversible accommodation of Na-ions.

Previous reports indicate that the stacking thickness plays a critical role in the reactivity of radical intermediates. ${ }^{77,78} \mathrm{Lu}$ and coworkers utilized DAAQ-COF and exfoliated it to varying thicknesses to investigate its effect on COF-based anode materials in SIBs (Figure 22a-c). ${ }^{79}$ The four sample thicknesses obtained after ball milling at various frequencies were $100-250,100-180,50-85$, and $4-12 \mathrm{~nm}$. The charge/ discharge voltage profiles of the samples exhibit two discharge plateaus correlating to two Na-ion insertion processes (Figure 22d). Cycling performances were studied at a rate of $100 \mathrm{~mA} \mathrm{~g}^{-1}$ in the voltage range of $0.5-3.0 \mathrm{~V}$ (Figure 22e). After an initial activation period, reversible a)

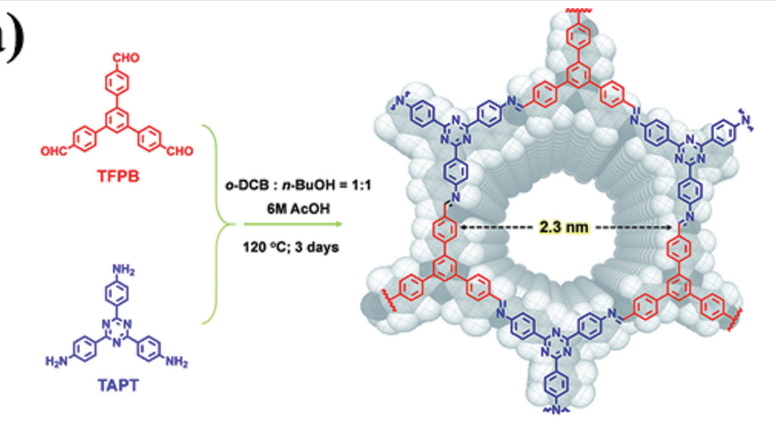

TFPB-TAPT COF

b)

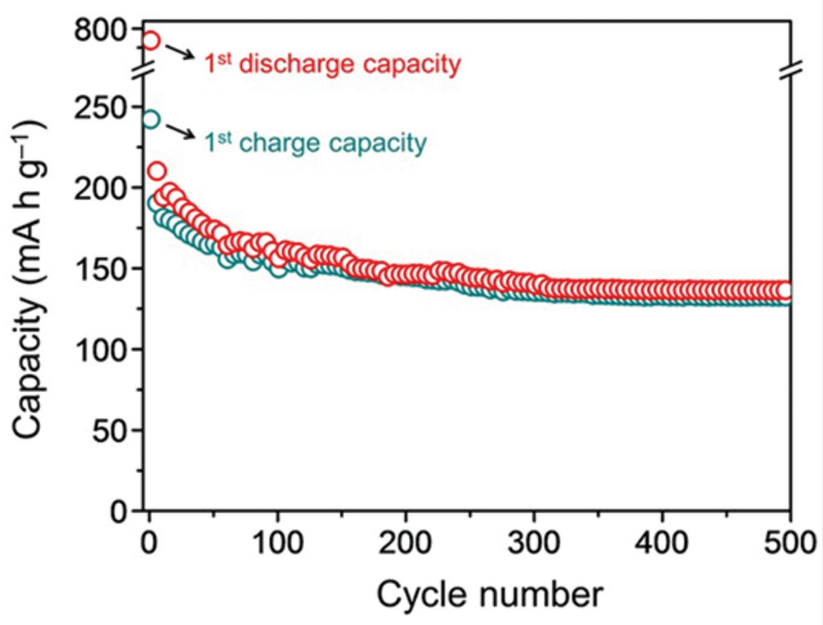

Figure 21 (a) Synthesis of TFPB-TAPT-COF. (b) Cycling performance of TFPB-TAPT COF at $30 \mathrm{~mA} \mathrm{~g}^{-1}$. Adapted with permission from Ref. 76. Copyright 2018 The Royal Society of Chemistry.

capacities of $420,310,208$, and $154 \mathrm{~mA} \mathrm{~h} \mathrm{~g}^{-1}$ were observed for 4-12, 50-85, 100-180, and 100-250 nm COF thicknesses, respectively. Notably, the activation was found to vary based on COF thickness, stabilizing after 5, 10, 15, and 30 cycles when changing from the thinnest to thickest samples, respectively. These results indicate that weakening the stacking interaction by decreasing COF thickness may enhance both stability and capacity of the electrode. These weak stacking interactions may be reducing the electron selfexchange process and thus making more active sites available. The Nyquist plot confirmed that as COF layer thickness decreased, ionic conductivity of the electrode increased (Figure 22f). The subsequent investigation of rate performances at varying current densities also confirmed the benefit of reduced thickness on capacity and cycle stability (Figure 22g). The 4-12 nm sample displayed capacities of $500,450,420,390,350,320,310$, and $300 \mathrm{~mA} \mathrm{~h} \mathrm{~g}^{-1}$ at current densities of $50,75,100,150,200,400,500$, and $1000 \mathrm{mAg}^{-1}$, respectively. Conversely, the $100-250 \mathrm{~nm}$ sample exhibited 
a)

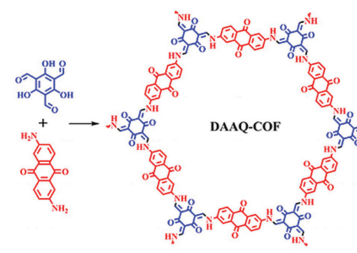

d)

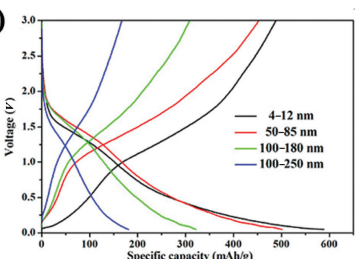

f)

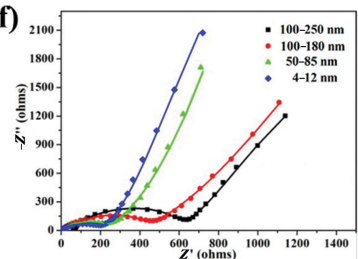

b)

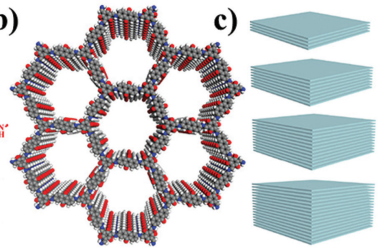

e)

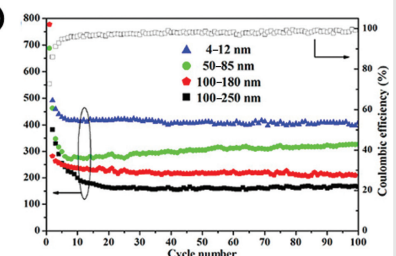

g)

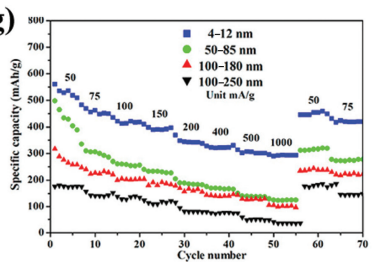

Figure 22 (a) Synthesis and (b) simulated structure of DAAQ-COF. (c) Representation of layer-by-layer stacking in a COF with different thicknesses. (d) Voltage profiles for the second cycle at $50 \mathrm{~mA} \mathrm{~g}^{-1}$. (e) Cycle performances of COFs with different thicknesses at $100 \mathrm{~mA} \mathrm{~g}^{-}$ and the coulombic efficiency of the 4-12 nm sample. (f) Nyquist plots of COFs with different thicknesses. $(\mathrm{g})$ Rate performances at various current densities between 50 and $1000 \mathrm{~mA} \mathrm{~g}^{-1}$. Adapted with permission from Ref. 79. Copyright 2019 American Chemical Society.

much lower capacities at the same current densities of 182 , $148,137,125,92,76$, and $47 \mathrm{~mA} \mathrm{~h} \mathrm{~g}^{-1}$, respectively. Furthermore, the $4-12 \mathrm{~nm}$ sample reached a reversible capacity of $198 \mathrm{~mA} \mathrm{~h} \mathrm{~g}^{-1}$ at a high current density of $5 \mathrm{~A}$ $\mathrm{g}^{-1}$, remaining stable for 10000 cycles with a capacity

retention of 99\%. Scanning electron microscopy (SEM) images also showed little difference in the morphologies before and after cycling, indicating the stability of the electrodes.

Designing COF-based cathode materials with high capacity and rate capability is a challenge partially due to the inactive groups that act as linkages in the COF skeleton. Chen and coworkers sought to eliminate this issue with the design of TQBQ-COF (Figure 23a). ${ }^{80}$ This COF forms via a condensation reaction between tetraminophenone and cyclohexanehexanone endowing the COF skeleton with triquinoxalinylene $(\mathrm{TQ})$ and benzoquinone $(\mathrm{BQ})$ units and no inactive linkage groups. The carbonyl and pyrazine groups in the COF skeleton can both be redox-active sites resulting in a theoretical capacity of $515 \mathrm{~mA} \mathrm{~h} \mathrm{~g}^{-1}$. Fourier transform infrared spectroscopy (FT-IR) was employed to examine the sodium-storage mechanism during the charge/discharge process for two cycles (Figure 23b, c). The Na-ion storage ability by carbonyls and pyrazine units in the COF skeleton could be confirmed by the corresponding peak changes in the charging process followed by the gradual recovery of the $\mathrm{C}=\mathrm{O}$ and $\mathrm{C}=\mathrm{N}$ peaks during the discharge process. This could be observed again during the second cycle indicating the reversibility of the sodiation/desodiation process. Ex situ XPS data were in good agreement with the FT-IR results (Figure 23d), further confirming the role of both carbonyl and pyrazine sites in the sodium-storage mechanism. Density functional theory calculations were utilized to gain insight into the sodiation/desodiation process wherein it was determined that the first six $\mathrm{Na}$-ions will each be accommodated between two adjacent nitrogen atoms. The next six Na-ions are each located outside the TQBQ-COF plane between two adjacent oxygen and nitrogen atoms for a total of 12 ions involved in the intercalation/deintercalation process a)

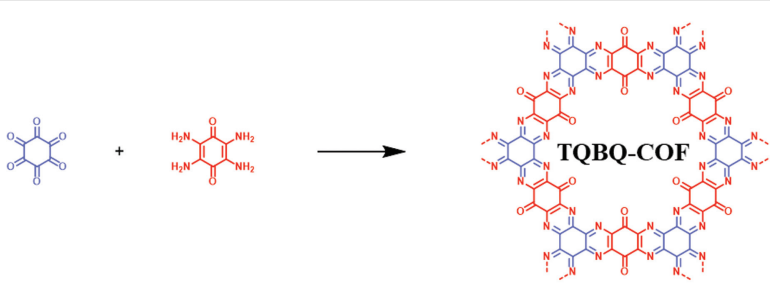

e)

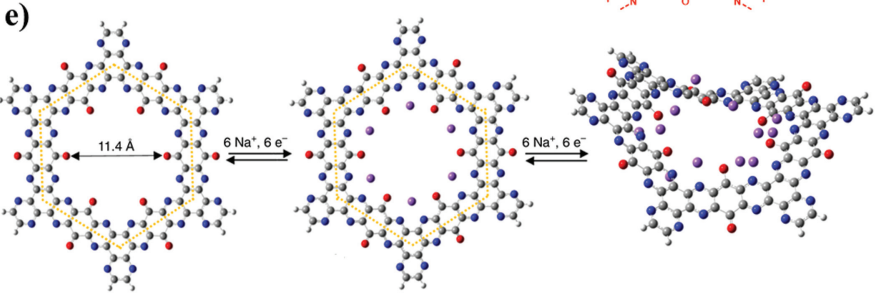

b)

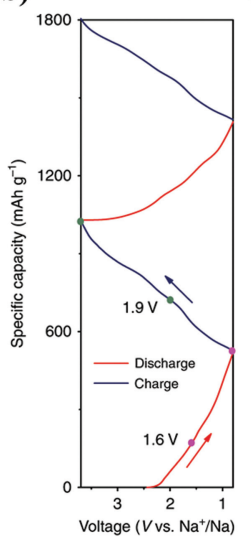

c)

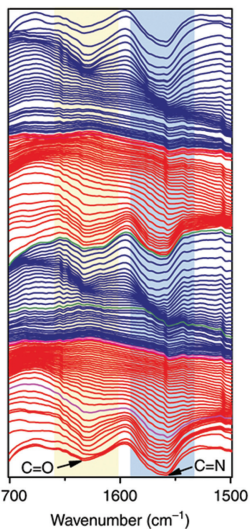

d)

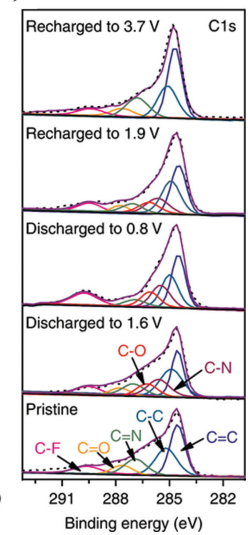

Figure 23 (a) Synthesis of TQBQ-COF. (b) Charge/discharge profile of TQBQ-COF in the first two cycles at $0.02 \mathrm{~A} \mathrm{~g}^{-1}$ in the voltage range of $0.8-3.7 \mathrm{~V}$. (c) In situ FT-IR spectra at different states corresponding to (b). (d) C1s XPS spectra of TQBQ-COF electrodes at different states corresponding to (b). (e) Schematic diagram of the two-step sodiation/desodiation process in TQBQ-COF obtained via the molecular electrostatic potential (MESP) method. Adapted with permission from Ref. 80. Copyright 2020 Springer Nature. 
(Figure 23e). The galvanostatic charge/discharge profile at $0.02 \mathrm{~A} \mathrm{~g}^{-1}$ from 1 to $3.6 \mathrm{~V}$ exhibited an initial discharge capacity of $452 \mathrm{~mA} \mathrm{~h} \mathrm{~g}^{-1}$ and a reversible capacity of about $400 \mathrm{~mA} \mathrm{~h} \mathrm{~g}{ }^{-1}$ after 10 cycles and $352 \mathrm{~mA} \mathrm{~h} \mathrm{~g}^{-1}$ after 100 . After 1000 cycles at 0.5 and $1.0 \mathrm{~A} \mathrm{~g}^{-1}$, the electrode displayed reversible capacities of 236.5 and $213.6 \mathrm{~mA} \mathrm{~h}^{-1}$, respectively. Columbic and energy efficiencies were around $100 \%$ and $85 \%$, respectively, indicating good stability and high-energy efficiency. The high rate performance was ascribed to the morphology of TQBQ-COF, which allows for the rapid ion/electron transport among the multiple redox sites available.

In comparison to Li-ions, Na-ions are larger and therefore exhibit lower ionic mobility in battery applications. There have been reports of doping hard carbon with heteroatoms such as $\mathrm{B},{ }^{81} \mathrm{~S},{ }^{82} \mathrm{~N},{ }^{83}$ and $\mathrm{P}^{84}$ in efforts to reduce the sluggishness of $\mathrm{Na}$-ions by introducing a chemical driving force. While these efforts have seen success, rate performance remains an issue. Vaidhyanathan and coworkers synthesized three mesoporous COFs to use as anodes in SIBs. IISERPCOF16, IISERP-COF17, and IISERP-COF18 were synthesized containing phenyl, tetrazine, and bispyridine-tetrazine moieties, respectively, in efforts to drive Na-ions into their pores via favorable interactions (Figure 24a). ${ }^{85} \mathrm{CVs}$ were used to investigate the sodiation/desodiation mechanisms within a potential window of 0.05-3 V (Figure 24c). For IISERP-COF17 and IISERP-COF18, the sodium insertion during the discharge occurred via a two-step process (Figure 24b). IISERP-COF16, the only COF possessing no $\pi$-stacked $s$-tetrazine rings through the nanochannel, displayed very little current output. During the discharge process, the anode becomes negatively charged. The electron-deficient $s$-tetrazine rings in IISERPCOF17 and IISERP-COF18 are able to accommodate the influx of electrons, favorably impacting the $\mathrm{Na}$-ion insertion into the nanopores of the COFs. The ease of the reduction of the anodic COF is thought to depend on the stabilization of the LUMO energy level (Figure 24d). The tetrazine units in IISERP-COF18 are conjugated with a pyridine ring, lowering the LUMO in comparison to IISERP-COF17. IISERP-COF18 exhibited the highest specific capacity of $410 \mathrm{~mA} \mathrm{~h} \mathrm{~g}^{-1}$ at a current density of $100 \mathrm{~mA} \mathrm{~g}^{-1}$ (Figure 24e). The anodic COFs displayed high rate performances, maintaining about $80 \%$ of their specific capacities at a current density of $1 \mathrm{Ag}^{-1}$ (Figure 24f). IISERPCOF17 and IISERP-COF18 both showed high electrochemical stability, while IISERP-COF16 lost most of its specific capacity at $500 \mathrm{~mA} \mathrm{~g}^{-1}$, further indicating the important role the tetrazine units play in accommodating high electron densities.

Potassium-ion batteries (PIBs) have been suggested as an alternative energy-storage system due to abundant resources, low electrolyte cost, and weaker Lewis acidity. ${ }^{86}$ Hazardous potassium dendrites can form during the charge/discharge process making choice of anode material critical. In 2019, Wang and coworkers reported the controlled growth of COF-10 on a CNT (COF-10@CNT) surface to use as an anode material in PIBs. ${ }^{87}$ COF-10 was synthesized via the polycondensation of 4,4'-biphenyldiboronic acid and TP (Figure 25a, b). The charge/discharge profile of COF-10@CNT showed an initial capacity of $348 \mathrm{~mA} \mathrm{~h} \mathrm{~g}^{-1}$ while COF-10 showed a lower initial capacity of $130 \mathrm{~mA} \mathrm{~h} \mathrm{~g}{ }^{-1}$ at a current loading of $0.1 \mathrm{~A} \mathrm{~g}^{-1}$ (Figure 25c). COF-10@CNT exhibited a stable cycling performance indicating a robust electrode structure and reversible a)

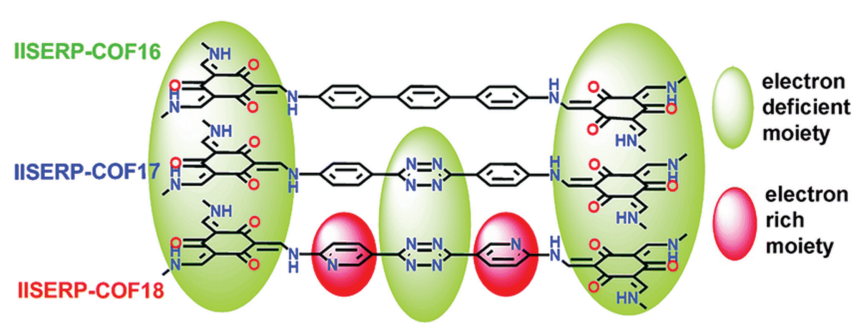

b)

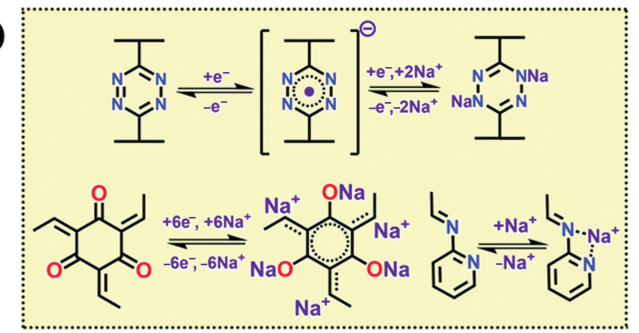

c)

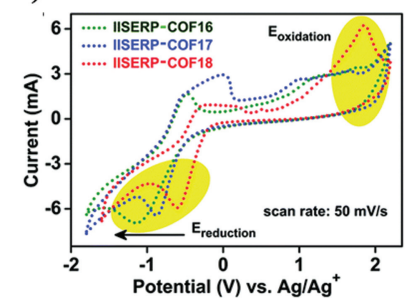

d)

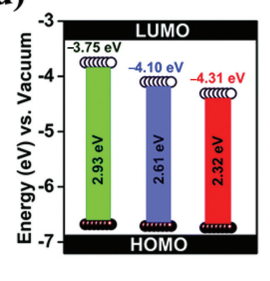

e)

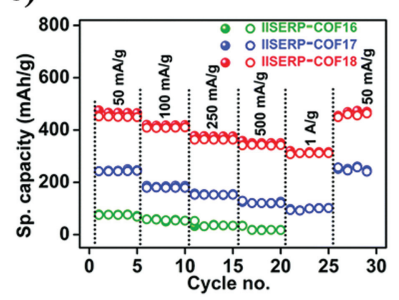

f)

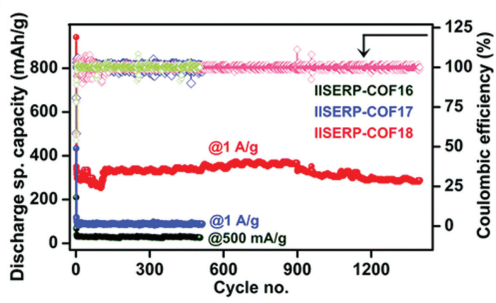

Figure 24 (a) Building blocks of the three COFs showing the presence of electron-deficient and electron-rich moieties. (b) Electrochemical reduction mechanism of (top) tetrazine and (bottom) phloroglucinol, followed by sodiation under a withdrawing potential. (c) CV curves of COFs. (d) HOMOLUMO energy levels and band gaps of the COFs calculated from the CV measurements. (e) Rate performance of COFs. (f) Cycling stability of the COFs at 1 $\mathrm{A} \mathrm{g}^{-1}$. Adapted with permission from Ref. 85. Copyright 2020 The Royal Society of Chemistry. 
a)
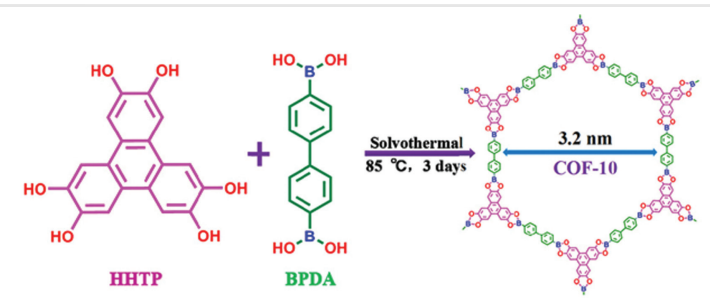

b)

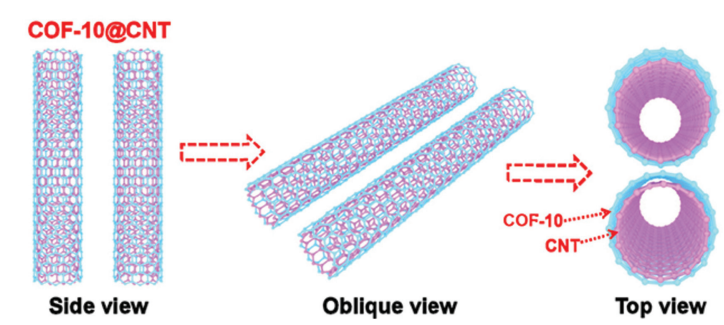

c)

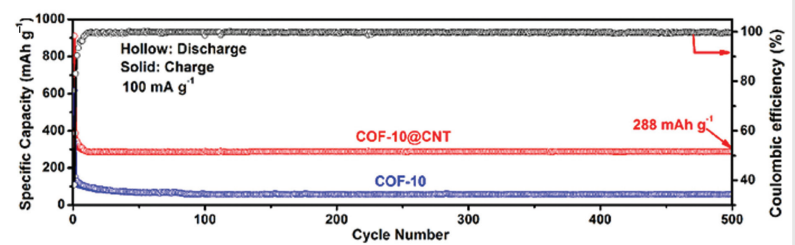

d)
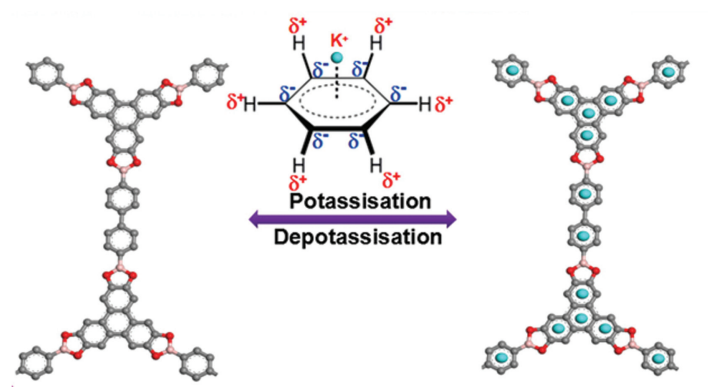

Figure 25 (a) Synthesis of COF-10. (b) Schematic representation of COF-10@CNT. (c) Cycling performance of COF-10 and COF-10@CNT at $100 \mathrm{~mA} \mathrm{~g}^{-1}$. (d) Representation of proposed potassium storage mechanism. Adapted with permission from Ref. 87. Copyright 2019 American Chemical Society.

electrochemical properties. After 500 cycles, COF-10@CNT maintained a reversible capacity of $288 \mathrm{~mA} \mathrm{~h} \mathrm{~g} \mathrm{~g}^{-1}$ outperforming previous reports of organic anodes for PIBs. ${ }^{88-92}$ The porous few-layered COF-10@CNT provided more exposed active sites, shortens the ion/electron diffusion distance, and facilitates the kinetics of the potassiation/depotassiation process. The potassium-storage mechanism (Figure 25d), investigated using XPS and Raman spectroscopy, is likely influenced by the $\pi$-cation effect between the $\pi$-electron clouds of benzene and K-ions.

In 2020, Alshareef, Eddaoudi, and coworkers reported the synthesis of PA-COF, a phenanthroline-based COF to use as a zinc-ion supercapattery (ZIS) cathode (Figure 26a). ${ }^{93}$ ZISs a)

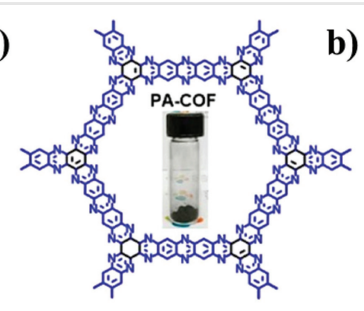

b)

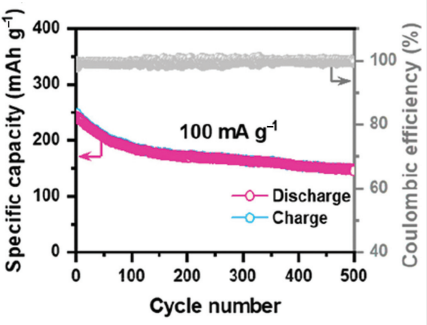

c)

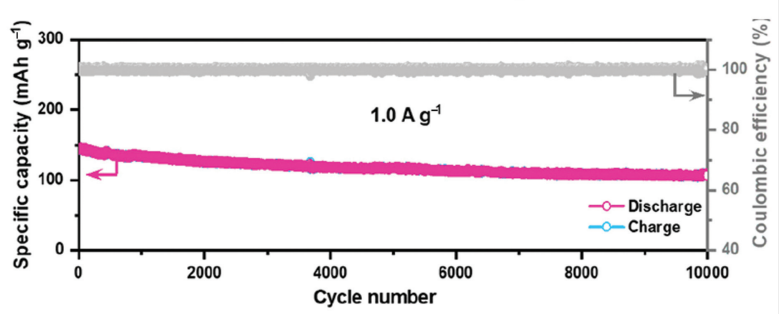

d)

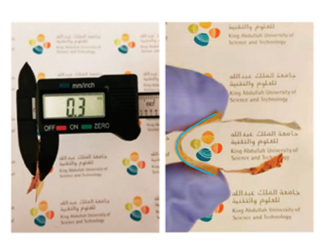

e)

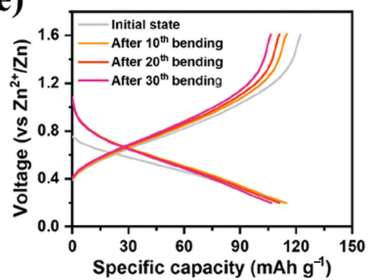

Figure 26 (a) Structure of PA-COF. (b) Cycling performance at $100 \mathrm{~mA}$ $\mathrm{g}^{-1}$. (c) Cycling stability at $1 \mathrm{~A} \mathrm{~g}^{-1}$. (d) Thickness and bending measurements of flexible supercapattery using PA-COF electrodes. (e) Charge/discharge curves at initial state and after bending the flexible supercapattery. Adapted with permission from Ref. 93. Copyright 2020 American Chemical Society.

represent yet another class of promising energy-storage devices due to their nontoxicity and relative inexpensiveness. ${ }^{94}$ In 2019, Shao and coworkers found that nitrogen substitution in 1,4,5,8-naphthalenetetracarboxylic dianhydride yielding 1,4,5,8-naphthalene diimide led to an electrode with improved specific capacity and cycling stability. ${ }^{95}$ $\mathrm{N}$-doping of porous carbon was also shown to promote the chemical adsorption of Zn-ions by Lu and coworkers. ${ }^{96}$ It was hypothesized that PA-COF, containing an electron-rich backbone and nitrogen active sites, would facilitate the reversible $\mathrm{Zn}$-ion intercalation. To evaluate the material's electrochemical performance, PA-COF was utilized as a cathode. PA-COF exhibited a high capacity of $247 \mathrm{~mA} \mathrm{~h} \mathrm{~g}^{-1}$ at a current loading of $0.1 \mathrm{~A} \mathrm{~g}^{-1}$ (Figure 26b). The material was able to maintain a capacity of $175 \mathrm{~mA} \mathrm{~h}^{-1}$ after 300 cycles at a current density of $0.1 \mathrm{Ag}^{-1}$, demonstrating its cycling performance. The material further demonstrated its high cycling stability, achieving a capacity decay rate of $0.38 \%$ per cycle over 10000 cycles at a current density of $1 \mathrm{~A} \mathrm{~g}^{-1}$ (Figure 26c). The high capacity was attributed to the chemical intercalation of ions coupled with electron transfer on the active sites of the COF. The authors used various physiochemical techniques to confirm the intercalation/deintercalation of $\mathrm{Zn}$-ions through the PA-COF electrode. 
Interestingly, through solid-state NMR, the authors were able to determine that the cointercalation of $\mathrm{Zn}$-ions and $\mathrm{H}$-ions demonstrated capacity contributions of $60 \%$ and $40 \%$, respectively. Furthermore, the authors used PA-COF to design and fabricate a high-performance supercapattery for practical applications (Figure 26d). The flexible device demonstrated high stability while being bent and produced similar charge/discharge profiles (Figure 26e), indicating its promising future in energy-storage and wearable electronics.

\section{Conclusions and Outlook}

In this review, it was shown that the unique design and electronic properties of COFs give them a unique advantage as rechargeable battery electrode materials for not only traditional LIBs, but also less established battery technologies including Li-S batteries and PIBs. However, the development of COF-based electrode materials is still in its infancy, and further improvement will require addressing performance issues such as poor conductivity and capacity fading at high rates in bulk form.

Currently, the largest bottleneck in COF-based electrode performance remains their poor electronic conductivity. Investigation of COF-based composites on highly conductive substrates has been done in an attempt to alleviate this issue. However, in an ideal scenario, highly conductive COFbased electrode materials would allow for the exclusion of all conductive carbon additives, which would increase the overall energy density of the cell. Currently, it is still necessary to include these sources because COFs are not conductive enough to facilitate charge through their bulk structure from/to the current collector. Overcoming this problem will require further development of COF-based materials with high degrees of conjugation without the addition of redox-inactive building units. It is also understood that COF stacking patterns can affect the out-of-plane conductivity of electronic systems. Presently, it is unclear what role this plays in tuning the high-rate performance of COF-based electrode materials. Therefore, further experimental and theoretical investigations are warranted.

Poor ionic conductivity through bulk materials also remains an issue negatively affecting performance. Processing methods involving exfoliation into thin sheets has proven to be a powerful solution to this issue. However, it remains advantageous to leverage the modularity of COF synthesis to incorporate moieties that improve ionic conductivity without sacrificing either capacity or electronic conductivity. In order to achieve this, further investigation into ion conduction in COF structures will be important. Computational modeling may prove very useful in determining moieties best suited for particular ion systems.

COFs are a highly versatile class of materials amenable for use in a multitude of applications, and recent progress proves that advanced electrode materials are no exception. Their variable design strategies offer limitless architectures, which can be assembled with molecular precision, allowing for unprecedented control over energy-storage mechanisms. However, these properties can only be leveraged through the development and investigation of novel systems. This will require expanding beyond the limited number of building blocks that have been utilized to construct COFs. Even more so, it will be advantageous to expand the current library of reversible linkages shown to produce ordered COF structures. Additionally, investigating the effects of transitioning from 2D to 3D COF architectures for energy-storage applications remains unexplored. While it is likely that this change would reduce conductivity throughout the material, there may be distinct benefits gained with regards to charge kinetics and active-site accessibility. Particularly, if COF crystallization results in a bor topology, very high solvent permeability can be expected in addition to access to active sites that would be unhindered by adjacent layers as opposed to $2 \mathrm{D}$ systems or interpenetrated 3D systems. It is unknown whether these properties would improve or diminish their performance, overall.

In summary, significant progress has been made in utilizing COFs as an advanced class of electrode material. As a direct result, high-performing batteries have been presented using COF-based materials as both anodes and cathodes with exceptional capacities. With more investigations into structure-property relationships and the synthesis of other novel porous polymeric systems on the horizon, we believe that COFs will undoubtedly achieve their full potential as electrode materials for rechargeable batteries in due time.

\section{Funding Information}

We acknowledge financial support from the National Science Foundation (CBET-2037707).

\section{References}

(1) Kim, T.; Song, W.; Son, D.; Ono, L. K.; Qi, Y.J. Mater. Chem. A 2019, 7, 2942.

(2) Myung, S.; Maglia, F.; Park, K.; Yoon, C. S.; Lamp, P.; Kim, S.; Sun, Y. ACS Energy Lett. 2017, 2, 196.

(3) Kim,J.H.; Pieczonka, N.P.W.; Yang, L. ChemPhysChem 2014, 15, 1940.

(4) Schon, T. B.; McAllister, B. T.; Li, P. F.; Seferos, D. S. Chem. Soc. Rev. 2016, 45, 6345.

(5) Delaporte, N.; Lajoie, G.; Collin-Martin, S.; Zaghib, K. Sci. Rep. 2020, 10, 3812.

(6) Hu, L.; Wu, H.; La Mantia, F.; Yang, Y.; Cui, Y.ACS Nano 2010, 4, 5843.

(7) Nyholm, L.; Nyström, G.; Mihranyan, A.; Strømme, M. Adv. Mater. 2011, 23, 3751.

(8) Kim, T. S.; Lim, J.; Oh, M.; Kim, J. J. Power Sources 2017, 361, 15.

(9) Visco, S. J.; Liu, M.; Armand, M. B.; de Jonghe, L. C. Mol. Cryst. Liq. Cryst. 1990, 190, 185. 
(10) Song, Z.; Zhan, H.; Zhou, Y. Chem. Commun. 2009, 448.

(11) Geng, K.; He, T.; Liu, R.; Dalapati, S.; Tan, K. T.; Li, Z.; Tao, S.; Gong, Y.; Jiang, Q.; Jiang, D. Chem. Rev. 2020, 120, 8814.

(12) Côté, A. P.; Benin, A. I.; Ockwig, N. W.; O'Keeffe, M.; Matzger, A. J.; Yaghi, O. M. Science 2005, 310, 1166.

(13) Wang, H.; Wang, H.; Wang, Z.; Tang, L.; Zeng, G.; Xu, P.; Chen, M.; Xiong, T.; Zhou, C.; Li, X.; Huang, D.; Zhu, Y.; Wang, Z.; Tang, J. Chem. Soc. Rev. 2020, 49, 4135.

(14) Sharma, R. K.; Yadav, P.; Yadav, M.; Gupta, R.; Rana, P.; Srivastava, A.; Zbořil, R.; Varma, R. S.; Antonietti, M.; Gawande, M. B. Mater. Horiz. 2020, 7, 411.

(15) Rogge, S. M.J.; Bavykina, A.; Hajek, J.; Garcia, H.; Olivos-Suarez, A. I.; Sepúlveda-Escribano, A.; Vimont, A.; Clet, G.; Bazin, P.; Kapteijn, F.; Daturi, M.; Ramos-Fernandez, E. V.; Llabrés I Xamena, F. X.; Van Speybroeck, V.; Gascon, J. Chem. Soc. Rev. 2017, 46, 3134

(16) Ozdemir, J.; Mosleh, I.; Abolhassani, M.; Greenlee, L. F.; Beitle, R. R.; Beyzavi, M. H. Front. Energy Res. 2019, 7, 77.

(17) Haug, W. K.; Moscarello, E. M.; Wolfson, E. R.; McGrier, P. L. Chem. Soc. Rev. 2020, 49, 839

(18) Wu, C.; Liu, Y.; Liu, H.; Duan, C.; Pan, Q.; Zhu, J.; Hu, F.; Ma, X.; Jiu, T.; Li, Z.; Zhao, Y. J. Am. Chem. Soc. 2018, 140, 10016.

(19) Wang, M.; Guo, H.; Xue, R.; Li, Q.; Liu, H.; Wu, N.; Yao, W.; Yang, W. ChemElectroChem 2019, 6, 2984.

(20) Kim, T.; Song, W.; Son, D.; Ono, L. K.; Qi, Y.J. Mater. Chem. A 2019, 7, 2942.

(21) Baldwin, L. A.; Crowe, J. W.; Pyles, D. A.; McGrier, P. L.J. Am. Chem. Soc. 2016, 138, 15134.

(22) Evans, A. M.; Ryder, M. R.; Ji, W.; Strauss, M. J.; Corcos, A.; Vitaku, E.; Flanders, N. C.; Bisbey, R. P.; Dichtel, W. Faraday Discuss. 2021, 225, 226.

(23) Dalapati, S.; Jin, S.; Gao, J.; Xu, Y.; Nagai, A.; Jiang, D. J. Am. Chem. Soc. 2013, 135, 17310.

(24) Haug, W. K.; Wolfson, E. R.; Morman, B. T.; Thomas, C. M.; McGrier, P. L. J. Am. Chem. Soc. 2020, 142, 5521.

(25) Pyles, D. A.; Crowe, J. W.; Baldwin, L. A.; McGrier, P. L. ACS Macro Lett. 2016, 5, 1055.

(26) Pyles, D. A.; Coldren, W. H.; Eder, G. M.; Hadad, C. M.; McGrier, P. L. Chem. Sci. 2018, 9, 6417.

(27) Waller, P. J.; AlFaraj, Y. S.; Diercks, C. S.; Jarenwattananon, N. N.; Yaghi, O. M. J. Am. Chem. Soc. 2018, 140, 9099.

(28) Bi, S.; Thiruvengadam, P.; Wei, S.; Zhang, W.; Zhang, F.; Gao, L.; Xu, J.; Wu, D.; Chen, J. S.; Zhang, F. J. Am. Chem. Soc. 2020, 142, 11893.

(29) Acharjya, A.; Longworth-Dunbar, L.; Roeser, J.; Pachfule, P.; Thomas, A. J. Am. Chem. Soc. 2020, 142, 14033.

(30) Lyu, H.; Diercks, C. S.; Zhu, C.; Yaghi, O. M.J. Am. Chem. Soc. 2019, $141,6848$.

(31) Matsumoto, M.; Valentino, L.; Stiehl, G. M.; Balch, H. B.; Corcos, A. R.; Wang, F.; Ralph, D. C.; Mariñas, B. J.; Dichtel, W. R. Chem 2018, 4, 308.

(32) Dey, K.; Pal, M.; Rout, K. C.; Kunjattu H, S.; Das, A.; Mukherjee, R.; Kharul, U. K.; Banerjee, R. J. Am. Chem. Soc. 2017, 139, 13083.

(33) Yuan, S.; Li, X.; Zhu, J.; Zhang, G.; Van Puyvelde, P.; Van der Bruggen, B. Chem. Soc. Rev. 2019, 48, 2665.

(34) Liu, L. H.; Yang, C. X.; Yan, X. P. J. Chromatogr. A 2017, 1479, 137.

(35) Liu, Z.; Wang, H.; Ou, J.; Chen, L.; Ye, M. J. Hazard. Mater. 2018, $355,145$.

(36) Liu, X.; Lim, G. J. H.; Wang, Y.; Zhang, L.; Mullangi, D.; Wu, Y.; Zhao, D.; Ding, J.; Cheetham, A. K.; Wang, J. Chem. Eng. J. 2021, 403, 126333.

(37) Karak, S.; Kandambeth, S.; Biswal, B. P.; Sasmal, H. S.; Kumar, S.; Pachfule, P.; Banerjee, R. J. Am. Chem. Soc. 2017, 139, 1856.
(38) Peng, P.; Shi, L.; Huo, F.; Zhang, S.; Mi, C.; Cheng, Y.; Xiang, Z. ACS Nano 2019, 13, 878.

(39) Zhang, N.; Wang, T.; Wu, X.; Jiang, C.; Chen, F.; Bai, W.; Bai, R. RSC Adv. 2018, 8, 3803.

(40) Yao, J.; Liu, C.; Liu, X.; Guo, J.; Zhang, S.; Zheng, J.; Li, S. J. Membr. Sci. 2020, 601, 117864

(41) Yang, D.; Yao, Z.; Wu, D.; Zhang, Y.; Zhou, Z.; Bu, X. J. Mater. Chem. A 2016, 4, 18621.

(42) Wolfson, E. R.; Xiao, N.; Schkeryantz, L.; Haug, W. K.; Wu, Y.; McGrier, P. L. Mol. Syst. Des. Eng. 2020, 5, 97.

(43) Manthiram, A.; Chung, S. H.; Zu, C. Adv. Mater. 2015, 27, 1980.

(44) Yin, Y.; Xin, S.; Guo, Y.; Wan, L. Angew. Chem. Int. Ed. 2013, 52, 13186.

(45) Manthiram, A.; Fu, Y.; Chung, S. H.; Zu, C.; Su, Y. S. Chem. Rev. 2014, 114, 11751.

(46) Yang, Y.; Zheng, G.; Cui, Y. Chem. Soc. Rev. 2013, 42, 3018.

(47) Demir-Cakan, R.; Morcrette, M.; Nouar, F.; Davoisne, C.; Devic, T.; Gonbeau, D.; Dominko, R.; Serre, C.; Férey, G.; Tarascon, J. M. J. Am. Chem. Soc. 2011, 133, 16154.

(48) Fu, Y.; Manthiram, A. Chem. Mater. 2012, 24, 3081.

(49) Xu, G.; Ding, B.; Shen, L.; Nie, P.; Han, J.; Zhang, X. J. Mater. Chem. A 2013, 1, 4490.

(50) Ji, L.; Rao, M.; Zheng, H.; Zhang, L.; Li, Y.; Duan, W.; Guo, J.; Cairns, E. J.; Zhang, Y. J. Am. Chem. Soc. 2011, 133, 18522.

(51) Jayaprakash, N.; Shen, J.; Moganty, S. S.; Corona, A.; Archer, L. A. Angew. Chem. Int. Ed. 2011, 50, 5904.

(52) Xin, S.; Gu, L.; Zhao, N. H.; Yin, Y. X.; Zhou, L. J.; Guo, Y. G.; Wan, L. J. J. Am. Chem. Soc. 2012, 134, 18510.

(53) Xu, G.; Ding, B.; Nie, P.; Shen, L.; Dou, H.; Zhang, X. ACS Appl. Mater. Interfaces 2014, 6, 194.

(54) Liao, H.; Ding, H.; Li, B.; Ai, X.; Wang, C.J.Mater.Chem. A 2014,2, 8854.

(55) Liao, H.; Wang, H.; Ding, H.; Meng, X.; Xu, H.; Wang, B.; Ai, X.; Wang, C. J. Mater. Chem. A 2016, 4, 7416.

(56) Meng, Y.; Lin, G.; Ding, H.; Liao, H.; Wang, C. J. Mater. Chem. A 2018, 6, 17186

(57) Seh, Z. W.; Sun, Y.; Zhang, Q.; Cui, Y.Chem. Soc. Rev. 2016, 45, 5605.

(58) Chen, D.; Avestro, A. J.; Chen, Z.; Sun, J.; Wang, S.; Xiao, M.; Erno, Z.; Algaradah, M. M.; Nassar, M. S.; Amine, K.; Meng, Y.; Stoddart, J. F. Adv. Mater. 2015, 27, 2907.

(59) Sun, J.; Klechikov, A.; Moise, C.; Prodana, M.; Enachescu, M.; Talyzin, A. V. Angew. Chem. Int. Ed. 2018, 57, 1034.

(60) Xiao, Z.; Li, L.; Tang, Y.; Cheng, Z.; Pan, H.; Tian, D.; Wang, R. Energy Storage Mater. 2018, 12, 252.

(61) Chung, W. J.; Griebel, J.J.; Kim, E. T.; Yoon, H.; Simmonds, A. G.; Ji, H. J.; Dirlam, P. T.; Glass, R. S.; Wie, J. J.; Nguyen, N. A.; Guralnick, B. W.; Park, J.; Somogyi, A.; Theato, P.; Mackay, M. E.; Sung, Y. E.; Char, K.; Pyun, J. Nat. Chem. 2013, 5, 518.

(62) Royuela, S.; Almarza, J.; Mancheño, M. J.; Pérez-Flores, J. C.; Michel, E. G.; Ramos, M. M.; Zamora, F.; Ocón, P.; Segura, J. L. Chem. Eur. J. 2019, 25, 12394.

(63) Fan, H.; Peng, M.; Strauss, I.; Mundstock, A.; Meng, H.; Caro, J. J. Am. Chem. Soc. 2020, 142, 6872.

(64) Shinde, D. B.; Sheng, G.; Li, X.; Ostwal, M.; Emwas, A. H.; Huang, K. W.; Lai, Z. J. Am. Chem. Soc. 2018, 140, 14342.

(65) Wang, S.; Wang, Q.; Shao, P.; Han, Y.; Gao, X.; Ma, L.; Yuan, S.; Ma, X.; Zhou, J.; Feng, X.; Wang, B. J. Am. Chem. Soc. 2017, 139, 4258.

(66) Chen, X.; Wang, L.; Xu, Y.; Wu, F.; Sun, W.; Wang, Y.; Li, Y.; Chen, L.; Wang, L.; Zhang, X.; Vajtai, R.; Ajayan, P. M.; Nie, A.; Li, Q.; Vajtai, R. Adv. Mater. 2019, 31, 1901640.

(67) Haldar, S.; Roy, K.; Nandi, S.; Chakraborty, D.; Puthusseri, D.; Gawli, Y.; Ogale, S.; Vaidhyanathan, R. Adv. Energy Mater. 2018 8,1702170 . 
(68) Wang, Z.; Li, Y.; Liu, P.; Qi, Q.; Zhang, F.; Lu, G.; Zhao, X.; Huang, X. Nanoscale 2019, 11, 5330.

(69) Gonçalves, R. S. B.; de Oliveira, A. B. V.; Sindra, H. C.; Archanjo, B. S.; Mendoza, M. E.; Carneiro, L. S. A.; Buarque, C. D.; Esteves, P. M. ChemCatChem 2016, 8, 743.

(70) Thote, J.; Aiyappa, H. B.; Deshpande, A.; Díaz Díaz, D.; Kurungot, S.; Banerjee, R. Chem. Eur. J. 2014, 20, 15961.

(71) Wang, G.; Chandrasekhar, N.; Biswal, B. P.; Becker, D.; Paasch, S.; Brunner, E.; Addicoat, M.; Yu, M.; Berger, R.; Feng, X. A. Adv. Mater. 2019, 31, 1901478.

(72) Xu, F.; Jin, S.; Zhong, H.; Wu, D.; Yang, X.; Chen, X.; Wei, H.; Fu, R.; Jiang, D. Sci. Rep. 2015, 5, 8225.

(73) Ai, Q.; Fang, Q.; Liang, J.; Xu, X.; Zhai, T.; Gao, G.; Guo, H.; Han, G.; Ci, L.; Lou, J. Nano Energy 2020, 72, 104657.

(74) Jerng, S. E.; Chang, B.; Shin, H.; Kim, H.; Lee, T.; Char, K.; Choi, J. W. ACS Appl. Mater. Interfaces 2020, 12, 10597.

(75) Yang, Y.; Zheng, G.; Cui, Y. Chem. Soc. Rev. 2013, 42, 3018.

(76) Patra, B. C.; Das, S. K.; Ghosh, A.; Anish, R. K.; Moitra, P.; Addicoat, M.; Mitra, S.; Bhaumik, A.; Bhattacharya, S.; Pradhan, A. J. Mater. Chem. A 2018, 6, 16655.

(77) Kolek, M.; Otteny, F.; Schmidt, P.; Mück-Lichtenfeld, C.; Einholz, C.; Becking, J.; Schleicher, E.; Winter, M.; Bieker, P.; Esser, B. Energy Environ. Sci. 2017, 10, 2334.

(78) Sun, D.; Rosokha, S. V.; Kochi, J. K. J. Am. Chem. Soc. 2004, 126, 1388.

(79) Gu, S.; Wu, S.; Cao, L.; Li, M.; Qin, N.; Zhu, J.; Wang, Z.; Li, Y.; Li, Z.; Chen, J.; Lu, Z. J. Am. Chem. Soc. 2019, 141, 9623.

(80) Shi, R.; Liu, L.; Lu, Y.; Wang, C.; Li, Y.; Li, L.; Yan, Z.; Chen, J. Nat. Commun. 2020, 11, 178.

(81) Stadie, N. P.; Billeter, E.; Piveteau, L.; Kravchyk, K. V.; Döbeli, M.; Kovalenko, M. V. Chem. Mater. 2017, 29, 3211.
(82) Zou, G.; Wang, C.; Hou, H.; Wang, C.; Qiu, X.; Ji, X. Small 2017, 13 , 1700762.

(83) Dan, R.; Chen, W.; Xiao, Z.; Li, P.; Liu, M.; Chen, Z.; Yu, F. Energy Fuels 2020, 34, 3923.

(84) Hou, H.; Shao, L.; Zhang, Y.; Zou, G.; Chen, J.; Ji, X. Adv. Sci. 2017, 4, 1600243.

(85) Haldar, S.; Kaleeswaran, D.; Rase, D.; Roy, K.; Ogale, S.; Vaidhyanathan, R. Nanoscale Horiz. 2020, 5, 1264.

(86) Wang, N.; Chu, C.; Xu, X.; Du, Y.; Yang, J.; Bai, Z.; Dou, S. Adv. Energy Mater. 2018, 8, 1801888.

(87) Chen, X.; Zhang, H.; Ci, C.; Sun, W.; Wang, Y. ACS Nano 2019, 13, 3600.

(88) Lei, K.; Li, F.; Mu, C.; Wang, J.; Zhao, Q.; Chen, C.; Chen, J. Energy Environ. Sci. 2017, 10, 552.

(89) Li, C.; Deng, Q.; Tan, H.; Wang, C.; Fan, C.; Pei, J.; Cao, B.; Wang, Z.; Li, J. ACS Appl. Mater. Interfaces 2017, 9, 27414.

(90) Xue, Q.; Li, D.; Huang, Y.; Zhang, X.; Ye, Y.; Fan, E.; Li, L.; Wu, F.; Chen, R. J. Mater. Chem. A 2018, 6, 12559.

(91) Deng, Q.; Pei, J.; Fan, C.; Ma, J.; Cao, B.; Li, C.; Jin, Y.; Wang, L.; Li, J. Nano Energy 2017, 33, 350.

(92) Wang, C.; Tang, W.; Yao, Z.; Chen, Y.; Pei, J.; Fan, C. Org. Electron. 2018, 62, 536.

(93) Wang, W.; Kale, V. S.; Cao, Z.; Kandambeth, S.; Zhang, W.; Ming, J.; Parvatkar, P. T.; Abou-Hamad, E.; Shekhah, O.; Cavallo, L.; Eddaoudi, M.; Alshareef, H. N. ACS Energy Lett. 2020, 5, 2256.

(94) Ming, J.; Guo, J.; Xia, C.; Wang, W.; Alshareef, H. N. Mater. Sci. Eng., $R$ 2019, 135, 58.

(95) Wang, X.; Chen, L.; Lu, F.; Liu, J.; Chen, X.; Shao, G. ChemElectroChem 2019, 6, 3644.

(96) Zhang, H.; Liu, Q.; Fang, Y.; Teng, C.; Liu, X.; Fang, P.; Tong, Y.; Lu, X. Adv. Mater. 2019, 31, 1904948. 